\title{
Biomarkers of environmental manganese exposure and associations with childhood neurodevelopment: a systematic review and meta-analysis
}

Weiwei Liu ${ }^{1 \dagger}$, Yongjuan Xin ${ }^{1 \dagger}$, Qianwen Li ${ }^{1}$, Yanna Shang ${ }^{1}$, Zhiguang Ping ${ }^{1}$, Junxia Min², Catherine M. Cahill ${ }^{3}$, Jack T. Rogers ${ }^{3^{*}}$ and Fudi Wang ${ }^{1,2^{*}}$ (D)

\begin{abstract}
Background: Although prior studies showed a correlation between environmental manganese (Mn) exposure and neurodevelopmental disorders in children, the results have been inconclusive. There has yet been no consistent biomarker of environmental Mn exposure. Here, we summarized studies that investigated associations between manganese in biomarkers and childhood neurodevelopment and suggest a reliable biomarker.

Methods: We searched PubMed and Web of Science for potentially relevant articles published until December 31th 2019 in English. We also conducted a meta-analysis to quantify the effects of manganese exposure on Intelligence Quotient $(\mathrm{IQ})$ and the correlations of manganese in different indicators.

Results: Of 1754 citations identified, 55 studies with 13,388 subjects were included. Evidence from cohort studies found that higher manganese exposure had a negative effect on neurodevelopment, mostly influencing cognitive and motor skills in children under 6 years of age, as indicated by various metrics. Results from cross-sectional studies revealed that elevated $\mathrm{Mn}$ in hair (H-Mn) and drinking water (W-Mn), but not blood (B-Mn) or teeth (T-Mn), were associated with poorer cognitive and behavioral performance in children aged 6-18 years old. Of these crosssectional studies, most papers reported that the mean of $\mathrm{H}-\mathrm{Mn}$ was more than $0.55 \mu \mathrm{g} / \mathrm{g}$. The meta-analysis concerning $\mathrm{H}-\mathrm{Mn}$ suggested that a 10-fold increase in hair manganese was associated with a decrease of 2.51 points $(95 \%$ confidence interval $(\mathrm{Cl}),-4.58,-0.45)$ in Full Scale $\mathrm{IQ}$, while the meta-analysis of B-Mn and W-Mn generated no such significant effects. The pooled correlation analysis revealed that $\mathrm{H}-\mathrm{Mn}$ showed a more consistent correlation with W-Mn than B-Mn. Results regarding sex differences of manganese associations were inconsistent, although the preliminary meta-analysis found that higher W-Mn was associated with better Performance IQ only in boys, at a relatively low water manganese concentrations (most below $50 \mathrm{\mu g} / \mathrm{L}$ ).

(Continued on next page)
\end{abstract}

\footnotetext{
* Correspondence: jack.rogers@mgh.harvard.edu; fwang@zju.edu.cn

${ }^{\dagger}$ Weiwei Liu and Yongjuan Xin contributed equally to this work.

${ }^{3}$ Neurochemistry Laboratory, Department of Psychiatry-Neuroscience,

Massachusetts General Hospital and Harvard Medical School, Charlestown,

MA, USA

'Department of Nutrition, Precision Nutrition Innovation Center, School of

Public Health, Zhengzhou University, Zhengzhou, China

Full list of author information is available at the end of the article
}

(C) The Author(s). 2020 Open Access This article is licensed under a Creative Commons Attribution 4.0 International License, which permits use, sharing, adaptation, distribution and reproduction in any medium or format, as long as you give appropriate credit to the original author(s) and the source, provide a link to the Creative Commons licence, and indicate if changes were made. The images or other third party material in this article are included in the article's Creative Commons licence, unless indicated otherwise in a credit line to the material. If material is not included in the article's Creative Commons licence and your intended use is not permitted by statutory regulation or exceeds the permitted use, you will need to obtain permission directly from the copyright holder. To view a copy of this licence, visit http://creativecommons.org/licenses/by/4.0/ The Creative Commons Public Domain Dedication waiver (http://creativecommons.org/publicdomain/zero/1.0/) applies to the data made available in this article, unless otherwise stated in a credit line to the data. 
(Continued from previous page)

Conclusions: Higher manganese exposure is adversely associated with childhood neurodevelopment. Hair is the most reliable indicator of manganese exposure for children at 6-18 years of age. Analysis of the publications demonstrated sex differences in neurodevelopment upon manganese exposure, although a clear pattern has not yet been elucidated for this facet of our study.

Keywords: Manganese exposure, Biomarker, Cognitive function, Behavior, Motor

\section{Background}

Environmental metal exposure normally occurs in coexposure to multiple metals, such as lead, cadmium, arsenic, mercury, chromium and manganese. Among these metals, manganese $(\mathrm{Mn})$ is an essential trace element [1], but it is toxic, especially for brain functions, when abnormally deposition occurs in the body [2].

Growing interest has been recently generated to understand environmental manganese exposure in children [3, 4]. Meta-analysis about autism spectrum disorder (ASD) indicated that the mean difference in blood and hair manganese concentrations between ASD and control individuals was not significant [5]. In terms of neurocognitive development, these epidemiological studies had inconsistent conclusions across different biomarkers [6-9], which also left open the question as to whether there exists a useful biomarker for Mn exposure.

Evidence-based studies have also evaluated this association between manganese in hair and childhood IQ [10]. However, no comprehensive meta-analysis has been performed to examine $\mathrm{Mn}$ associations between different indicators and neurodevelopment. Thus, to the best of our knowledge, no meta-analysis has been performed regarding the putative correlation between such $\mathrm{Mn}$ indicators. Compared with cognition, the impacts of $\mathrm{Mn}$ on behavioral and motor development in children have been less evaluated, although Mn-related motor changes, such as in manganism, have been evaluated more extensively in occupational exposures [11, 12]. In addition, the potential for sex difference in the consequences of manganese exposure has also drawn attention, as there may be some differences between males and females in patterns of exposure, gastrointestinal absorption of chemicals, metabolism and detoxification [13].

To address these research gaps, the goal of this systematic review and meta-analysis has been to summarize and quantify the scientific evidence through different biomarkers or sources in order to obtain a clearer understanding of the exposure-response relationship between $\mathrm{Mn}$ indicators (biomarkers or environmental samples) and neurodevelopmental outcomes. In addition, we performed meta-analyses to seek a pooled correlation between $\mathrm{Mn}$ indicators (hair, blood and drinking water) and, here, suggested a potential biomarker for further epidemiologic studies of the toxic impact of $\mathrm{Mn}$ in childhood neurodevelopment. We also performed a preliminary meta-analysis to quantify the sex difference between manganese indicators and intelligence. Our conclusions provide useful suggestions for future public health studies, especially on the consequences of heavy metal exposures, such as Mn, towards human health.

\section{Methods}

\section{Search strategy and inclusion criteria}

Our study was conducted according to the Preferred Reporting Items for Systematic Reviews and MetaAnalysis (PRISMA) Statement. The completed PRISMA checklist is provided in Additional file 1. This systematic review protocol was registered with PROSPERO (CRD42020182284). Two investigators (authors W.L. and Y.X.) independently conducted a literature search in PubMed and Web of Science for studies published through December 31th 2019 in English, using the following search terms: ("manganese" or "manganism" or "manganese exposure") and ("children" or "child" or "infant" or "childhood" or "adolescents" or "early life" or "young" or "younger populations") and ("neurotoxicity" or "neuropsychological effects" or "neurodevelopmental outcomes" or "cognition" or "cognitive" or "intellectual function" or "intellectual impairment" or "intelligence quotient" or "IQ" or "memory" or "attention" or "mental" or "academic performance" or "hyperactivity" or "behavior" or "hyperactive behaviors" or "neurobehavior" or "motor" or "neuromotor"). In addition, the references included in relevant articles were searched for additional eligible publications.

Studies included in this systematic review had to meet the following criteria of being: (1) An original peer reviewed article; (2) A study of populations up to 18 years of age; (3) Manganese exposure was assessed through medicinal biomarkers (i.e. hair and blood) or environmental samples (i.e. drinking water); (4) A study of neurodevelopment derived from manganese exposure, including: cognitive, behavioral and/or motor changes; (5) Potential confounders were adjusted in the mathematical model for the estimated association between $\mathrm{Mn}$ indicator and a specific neurological outcome in children.

For inclusion in the meta-analysis, studies had to satisfy the above criteria and had to have measured the 
effect of manganese exposure on neurodevelopment by regression models, while for correlation analysis, the correlation coefficient $(r)$ was provided. We excluded studies about attention deficit hyperactivity disorder (ADHD), which was reviewed in a recent paper, and the results of which showed higher peripheral manganese concentrations in children diagnosed with ADHD than those in controls [14]. We did not exclude articles published using the same population with different neurodevelopmental assessments $[15,16]$.

\section{Data extraction and quality assessment}

The following information was extracted by two investigators (W.L. and Y.X.) independently using a standardized data collection form: first author, publication year, biomarker, country/study name, study design, sample size, age, sources of manganese exposure, neurological assessments and neurodevelopmental outcomes. For meta-analysis, the regression coefficient $(\beta)$ with its $95 \%$ confidence interval $(\mathrm{CI})$ and correlation coefficient $(r)$ were also extracted. In the event of multiple articles published using the same population when assessing neurodevelopmental outcomes at different ages, and the same data were used in more than one publication, we consistently selected the most informative article, which was usually the most recent publication.

The guideline for Strengthening the Reporting of Observational Studies in Epidemiology (STROBE) was applied to assess the methodological quality of each study by two investigators (W.L. and Y.X.) independently [17]. The STROBE Statement is a checklist of 22 items that was initially developed to evaluate the systematic clarity in communicating research results in observational studies. This checklist has been used in systematic reviews to evaluate the methodological quality of observational studies [10, 18]. Nine items in methods (items 4-12) were selected, which covering the different aspects of methodology in observational studies. The methodological quality was classified by the number of items that the research met. To be more specific, articles that met 0-3 items, 4-6 items and 7-9 items were regarded as low, moderate and high methodological quality, respectively. Any disagreements were resolved by group discussion with a third investigator (Q.L.).

\section{Statistical analysis}

A regression coefficient $(\beta)$ with corresponding 95\% CI was used as the common measure of association across studies $[6-8,16,19-21]$. A study that stratified by sex was treated as two separate reports [20]. We used a random-effects model to calculate the summarized $\beta$ metrics and their corresponding 95\% CIs. The metaanalysis was restricted to studies that used the Wechsler scales to evaluate IQ and linear regression models to examine the relationships between manganese exposure and children's IQ scores. One study exhibited the scores of estimated IQ, vocabulary, block design and digit span, which were subtests from the Wechsler Intelligence Scale [22]. We took the scores of estimated IQ, block design and vocabulary as Full Scale IQ, Performance IQ and Verbal IQ, respectively [23].

Three manganese exposure metrics were included: hair, blood and drinking water. Furthermore, the $\beta$ metric was estimated through different expressions of manganese concentration: $\log _{10}, \log _{2}, \log _{e}$ or nontransformation. We unified the expression as a $\log _{10^{-}}$ transformation to mean that the change in IQ $(\beta)$ was associated with a 10-fold increase in the manganese exposure indicator, while we did not transform the $\beta$ in blood, which was transformed using $\log _{e}$ consistently.

More specifically, in a linear regression model where the manganese concentration $(x)$ was transformed by logarithm base 2 to correct the skewness of the data distributions, we expressed it into a $\log _{10}$-transformation by the formula $\log _{2} x * \beta=\log _{10} x * \beta 1$ to obtain a new coefficient $(\beta 1)$. The $\beta 1$ was approximately equal to $3.32 * \beta$. Two studies assessed the effect of manganese exposure with raw manganese concentration. We used the similar formula to transform it into the changes in base $\log _{10}$. Clearly, the $\beta 1$ was equal to $\mathrm{E}(x)^{*} \beta$. $\mathrm{E}(x)$ was a function about the mean of manganese concentration $(x)$, more specifically, $\mathrm{E}(x)=x / \log _{10} x$.

In addition, a meta-analysis of correlation coefficients was also performed. Firstly, the Fisher's $z$ transformation was used to transform data as below,

$$
\begin{aligned}
& \text { Fisher's } z=0.5 * \operatorname{In} \frac{1+r}{1-r} \\
& \mathrm{SE}=\sqrt{\frac{1}{n-3}}(n \text { is the size of the sample }) \\
& \text { summary } r=\frac{e^{2 z}-1}{e^{2 z}+1}(z \text { was the summary Fisher's } z)
\end{aligned}
$$

Then, we put the Fisher's $z$ and Standard Error (SE) into RevMan 5.3 using the generic inverse variance random effects model to obtain the summary Fisher's $z$. Finally, the formula 3 was used to estimate the summary $r$ [24].

The meta-analysis was performed using Stata version 14 for regression and RevMan 5.3 for correlation. Heterogeneity among studies was estimated using the $I^{2}$ statistic [25]. A "leave-one-out" sensitivity analysis and subgroup analysis based on the source of exposure were also performed. Publication bias was assessed using Egger's test with a significant value set to $p<0.10$ [26]. Except where noted otherwise, differences with a $p$-value $<0.05$ were considered significant. 


\section{Results}

A total of 1754 potentially relevant studies were identified through database searches (see Fig. 1). After applying the stringent inclusion and exclusion criteria described in the methods section, 55 original studies encompassing 13,388 children were ultimately included. Fifteen studies reporting 18 outcomes were included in the meta-analysis, with 9 studies for regression and 9 studies for correlation (see Fig. 1).

The sources of manganese exposure were mainly from industrial activities (i.e. metallurgy and mining) and drinking water (see Tables 1,2,3). More studies examined postpartum manganese exposure than prenatal exposure, meanwhile there were also some studies that measured manganese exposure from prenatal to postnatal periods. The concentrations of manganese were more frequently measured in biomarkers $(n=52$, i.e. hair, blood and teeth) than environmental samples $(n=21$, i.e. drinking water, particulate matter and soil). The associations between manganese in biomarkers and neurodevelopmental outcomes were investigated in 15 cohort studies and 37 cross-sectional studies (see Tables 1,2).
Table 3 presents the associations between manganese in drinking water (W-Mn) and neurodevelopment.

The neurological outcomes were assessed more frequently among children between 6 and 18 years of age than children under 6 years old. Amongst children under 6 years old, most studies were cohort studies with the different editions of Bayley Scales of Infant and Toddler Development applied to assess neurodevelopment, and the measurements of manganese mainly reflected prenatal exposures. In the older groups, the well-defined versions of Wechsler Intelligence Scale for Children were used to assess the children's general cognitive abilities. Specific cognitive functions were assessed through its subtests. For behavioral performance, the variant editions of Conners' Rating Scale were applied in most studies. For motor coordination, Finger Tapping Test and Luria Nebraska Motor Battery were administered in most studies. Among these studies, the most adjusted confounders in the mathematical model were maternal education, maternal intelligence, child age and sex, which were selected based on established or plausible associations with neurodevelopment. A large percentage

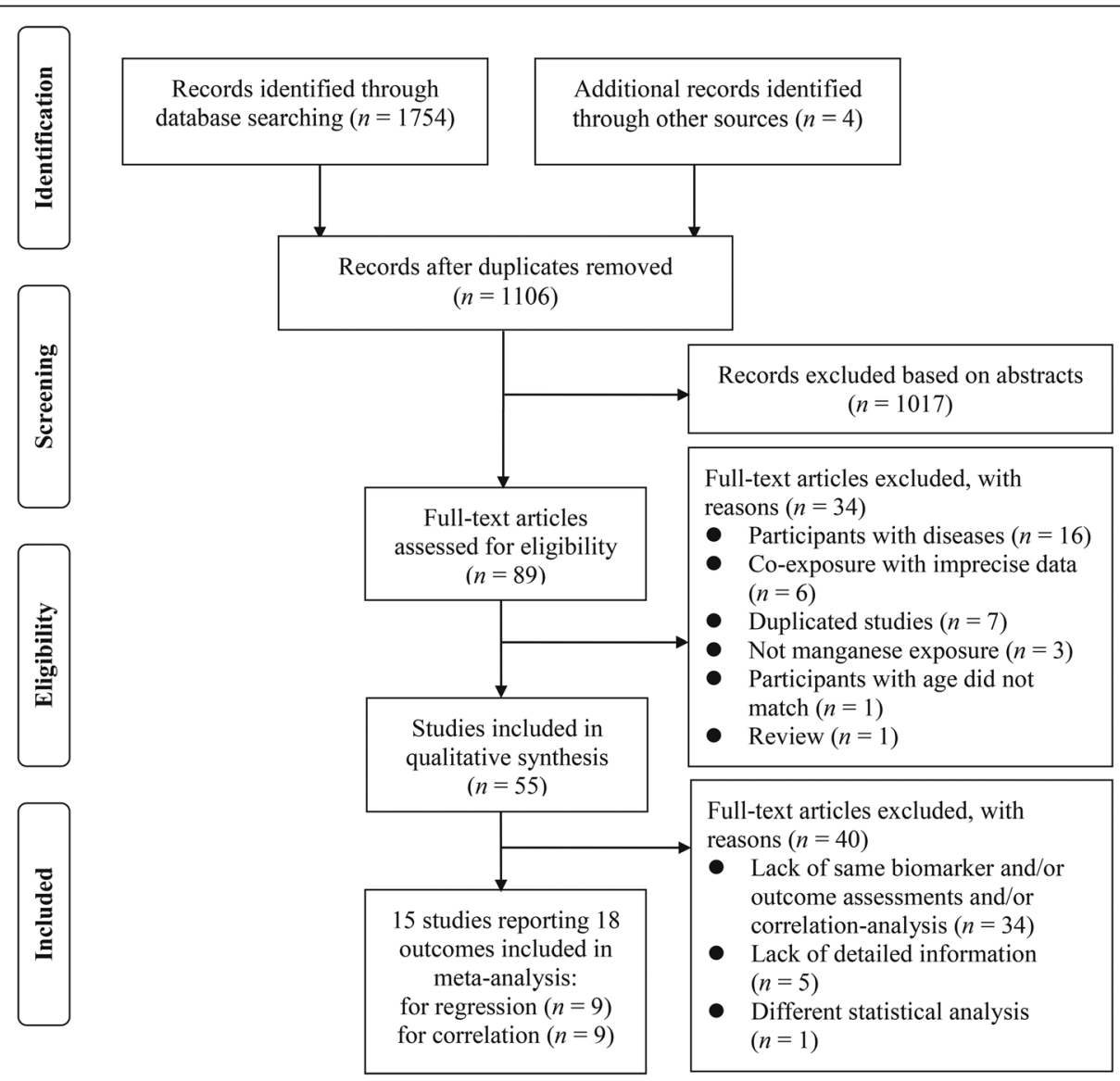

Fig. 1 PRISMA flow diagram 
Table 1 Neurodevelopmental outcomes of manganese exposure mainly prenatal exposure measured in biomarkers from cohort studies

\begin{tabular}{|c|c|c|c|c|c|c|c|c|c|}
\hline $\begin{array}{l}\text { Author, } \\
\text { Year }\end{array}$ & $\begin{array}{l}\begin{array}{l}\text { Age } \\
\text { (Years) }\end{array}\end{array}$ & $\begin{array}{l}\text { Country/ } \\
\text { Study } \\
\text { Name }\end{array}$ & $\begin{array}{l}\text { Number } \\
\text { (Girls/ } \\
\text { Boys) }\end{array}$ & Sources & Biomarkers & $\begin{array}{l}\text { Neurological } \\
\text { Assessments }\end{array}$ & $\begin{array}{l}\text { Associations between } \\
\text { Manganese in } \\
\text { Biomarkers and } \\
\text { Neurodevelopmental } \\
\text { Outcomes }\end{array}$ & $\begin{array}{l}\text { Adjustment for } \\
\text { Covariates }\end{array}$ & $\begin{array}{l}\text { Study } \\
\text { Quality }\end{array}$ \\
\hline $\begin{array}{l}\text { Chung } \\
2015 \text { [27] }\end{array}$ & 0.5 & $\begin{array}{l}\text { Korea/ } \\
\text { MOCEH }\end{array}$ & $\begin{array}{l}232(124 / \\
108)\end{array}$ & NA & $\begin{array}{l}\text { Maternal } \\
\text { blood }\end{array}$ & BSID-II & $\begin{array}{l}\text { An inverted U-shaped: } \\
\text { mental and psycho- } \\
\text { motor development }\end{array}$ & $\begin{array}{l}\text { Maternal age, } \\
\text { gestational age, } \\
\text { parity, income, } \\
\text { breastfeeding status, } \\
\text { maternal total } \\
\text { calorie intake, } \\
\text { residential area, } \\
\text { infant sex and birth } \\
\text { weight }\end{array}$ & High \\
\hline $\begin{array}{l}\text { Claus Henn } \\
2010 \text { [28] }\end{array}$ & $1-2$ & Mexico & $448^{\#}$ & NA & Blood & BSID-II & $\begin{array}{l}\text { An inverted U-shaped: } \\
\text { mental development } \\
\text { at } 1 \text { year of age, } 2 \text { years } \\
\text { of age: NS }\end{array}$ & $\begin{array}{l}\text { Blood lead, sex, } \\
\text { maternal IQ and } \\
\text { education, } \\
\text { hemoglobin and } \\
\text { gestational age }\end{array}$ & High \\
\hline $\begin{array}{l}\text { Claus Henn } \\
2017 \text { [29] }\end{array}$ & 2 & USA & $\begin{array}{l}224(91 / \\
133)\end{array}$ & Mining & $\begin{array}{l}\text { Maternal } \\
\text { blood, cord } \\
\text { blood }\end{array}$ & BSID-II & $\begin{array}{l}\text { Maternal blood: } \downarrow \text { : } \\
\text { mental and } \\
\text { psychomotor } \\
\text { development, cord } \\
\text { blood: NS }\end{array}$ & $\begin{array}{l}\text { Maternal age, } \\
\text { smoking, gestational } \\
\text { period, marital } \\
\text { status, parity, } \\
\text { income, and } \\
\text { prenatal vitamin use }\end{array}$ & High \\
\hline $\begin{array}{l}\text { Freire } 2018 \\
\text { [30] }\end{array}$ & $4-5$ & Spain & $\begin{array}{l}302(86 / \\
216)\end{array}$ & NA & Placenta & MSCA & $\begin{array}{l}\text { Placental Mn: } \downarrow \text { : } \\
\text { perceptual- } \\
\text { performance function, } \\
\uparrow: \text { memory span and } \\
\text { quantitative skills }\end{array}$ & $\begin{array}{l}\text { Child's sex, } \\
\text { psychologist, child } \\
\text { age, social class, } \\
\text { maternal smoking } \\
\text { during pregnancy } \\
\text { and pre-pregnancy } \\
\text { BMI }\end{array}$ & High \\
\hline $\begin{array}{l}\text { Gunier } \\
2015 \text { [31] }\end{array}$ & $0.5,1,2$ & $\begin{array}{l}\text { USA } \\
\text { CHAMACOS }\end{array}$ & $\begin{array}{l}197(113 / \\
84)\end{array}$ & NA & Teeth & BSID-\|I & $\begin{array}{l}\text { Postnatal T-Mn: } \downarrow \text { : } \\
\text { mental development } \\
\text { at 6-months and at 12- } \\
\text { months of age }\end{array}$ & $\begin{array}{l}\text { Child's age, sex, } \\
\text { maternal education, } \\
\text { IQ, psychometrician, } \\
\text { location of } \\
\text { assessment, } \\
\text { household poverty } \\
\text { and HOME score. } \\
\text { Postnatal models } \\
\text { also adjusted for } \\
\text { prenatal Mn }\end{array}$ & High \\
\hline $\begin{array}{l}\operatorname{Lin} 2013 \\
{[32]}\end{array}$ & 2 & China/TBPS & $\begin{array}{l}230(102 / \\
128)\end{array}$ & NA & Cord blood & CDIIT & $\begin{array}{l}\text { Cord blood: } \downarrow \text { : } \\
\text { cognition and } \\
\text { language }\end{array}$ & $\begin{array}{l}\text { Maternal age, } \\
\text { education, fish } \\
\text { intake, sex, passive } \\
\text { smoking and HOME } \\
\text { score }\end{array}$ & High \\
\hline $\begin{array}{l}\text { Mora } 2018 \\
\text { [33] }\end{array}$ & 1 & $\begin{array}{l}\text { Costa Rica/ } \\
\text { ISA }\end{array}$ & $\begin{array}{l}355(177 / \\
178)\end{array}$ & Mancozeb & $\begin{array}{l}\text { Maternal } \\
\text { blood and } \\
\text { hair }\end{array}$ & BSID-III & $\begin{array}{l}\text { Maternal hair: } \downarrow \text { : } \\
\text { cognition in girls, } \\
\text { maternal blood: NS }\end{array}$ & $\begin{array}{l}\text { Maternal education, } \\
\text { parity, gestational } \\
\text { period, child age, } \\
\text { HOME score and } \\
\text { location of } \\
\text { assessment }\end{array}$ & High \\
\hline $\begin{array}{l}\text { Takser } 2003 \\
{[34]}\end{array}$ & $0.7,3,6$ & France & $\begin{array}{l}195,126 \\
100(44 / \\
56)\end{array}$ & NA & * & MSCA & $\begin{array}{l}\text { Cord blood: } \downarrow \text { : } \\
\text { attention, non-verbal } \\
\text { memory, hand skill at } \\
3 \text { years old, the other } \\
\text { biomarkers: NS }\end{array}$ & $\begin{array}{l}\text { Child's sex and } \\
\text { mother's education }\end{array}$ & Medium \\
\hline $\begin{array}{l}\text { Yu } 2014 \\
{[35]}\end{array}$ & Newborns & China & $\begin{array}{l}933(439 / \\
494)\end{array}$ & NA & Cord serum & NBNA & $\begin{array}{l}\text { Cord serum Mn: } \downarrow \text { : fetal } \\
\text { neurobehavioral } \\
\text { development }\end{array}$ & $\begin{array}{l}\text { Maternal age, } \\
\text { education, } \\
\text { occupation, incomes, } \\
\text { birth weight, passive } \\
\text { smoking, gestational } \\
\text { age, sex, Pb and } \mathrm{Hg}\end{array}$ & High \\
\hline
\end{tabular}


Table 1 Neurodevelopmental outcomes of manganese exposure mainly prenatal exposure measured in biomarkers from cohort studies (Continued)

\begin{tabular}{|c|c|c|c|c|c|c|c|c|c|}
\hline $\begin{array}{l}\text { Author, } \\
\text { Year }\end{array}$ & $\begin{array}{l}\text { Age } \\
\text { (Years) }\end{array}$ & $\begin{array}{l}\text { Country/ } \\
\text { Study } \\
\text { Name }\end{array}$ & $\begin{array}{l}\text { Number } \\
\text { (Girls/ } \\
\text { Boys) }\end{array}$ & Sources & Biomarkers & $\begin{array}{l}\text { Neurological } \\
\text { Assessments }\end{array}$ & $\begin{array}{l}\text { Associations between } \\
\text { Manganese in } \\
\text { Biomarkers and } \\
\text { Neurodevelopmental } \\
\text { Outcomes }\end{array}$ & $\begin{array}{l}\text { Adjustment for } \\
\text { Covariates }\end{array}$ & $\begin{array}{l}\text { Study } \\
\text { Quality }\end{array}$ \\
\hline $\begin{array}{l}\text { Yu } 2016 \\
{[36]}\end{array}$ & 1 & $\begin{array}{l}\text { China/LW } \\
\text { birth cohort }\end{array}$ & $\begin{array}{l}377(188 / \\
189)\end{array}$ & NA & Cord serum & GDI & $\begin{array}{l}\text { Cord serum } \mathrm{Mn} \text { : } \downarrow \text { : } \\
\text { gross motor and } \\
\text { personal-social tasks }\end{array}$ & $\begin{array}{l}\text { Maternal education, } \\
\text { income, birth } \\
\text { weight, } \mathrm{Hg} \text { and Fe }\end{array}$ & High \\
\hline $\begin{array}{l}\text { Claus Henn } \\
2018[37]\end{array}$ & $6-16$ & $\begin{array}{l}\text { Mexico/ } \\
\text { ELEMENT }\end{array}$ & $\begin{array}{l}138(74 / \\
64)\end{array}$ & $\begin{array}{l}\text { Air } \\
\text { pollution } \\
\text { and diets }\end{array}$ & Teeth & WRAVMA & $\begin{array}{l}\text { NS, stratified by sex, } \\
\text { postnatal T-Mn: } \downarrow \text { : vis- } \\
\text { ual spatial scores in } \\
\text { boys only }\end{array}$ & $\begin{array}{l}\text { Child's sex, tooth Pb } \\
\text { levels, maternal IQ, } \\
\text { maternal education } \\
\text { and study cohort }\end{array}$ & High \\
\hline $\begin{array}{l}\text { Dion } 2018 \\
{[20]}\end{array}$ & $10.5-18$ & Canada & $\begin{array}{l}287(151 / \\
136)\end{array}$ & $\begin{array}{l}\text { Ground } \\
\text { water }\end{array}$ & Hair & WASI & $\begin{array}{l}\text { Hair: NS, water Mn } \\
\text { increased, Performance } \\
\text { IQ scores decreased }\end{array}$ & $\begin{array}{l}\text { Maternal IQ, } \\
\text { education and } \\
\text { income }\end{array}$ & High \\
\hline $\begin{array}{l}\text { Mora } 2015 \\
\text { [38] }\end{array}$ & $7,9,10.5$ & $\begin{array}{l}\text { USA } \\
\text { CHAMACOS }\end{array}$ & $\begin{array}{l}248(140 / \\
108)\end{array}$ & $\begin{array}{l}\text { Mancoze, } \\
\text { maneb }\end{array}$ & Teeth & $\begin{array}{l}\text { BASC-2 WISC- } \\
\text { IV }\end{array}$ & $\begin{array}{l}\text { Prenatal and postnatal } \\
\text { T-Mn: } \downarrow \text { : behavior in } \\
\text { boys and girls, } \uparrow: \text { motor, } \\
\text { memory and cognition } \\
\text { in boys }\end{array}$ & $\begin{array}{l}\text { Maternal education, } \\
\text { IQ, years in the US, } \\
\text { and depression at } \\
\text { time of assessment, } \\
\text { child's sex and age, } \\
\text { language of } \\
\text { maternal interview, } \\
\text { HOME score, income } \\
\text { and number of } \\
\text { children in the } \\
\text { home at time of } \\
\text { assessment }\end{array}$ & High \\
\hline $\begin{array}{l}\text { Wasserman } \\
2016 \text { [19] }\end{array}$ & $12.4 \pm 0.8$ & Bangladesh & 296 & $\begin{array}{l}\text { Deep well } \\
\text { water } \\
\text { with } \\
\text { reduced } \\
\text { Mn }\end{array}$ & Blood & WISC-IV & $\begin{array}{l}\text { Baseline B-Mn: } \downarrow \text { : work- } \\
\text { ing memory, reduc- } \\
\text { tions in B-Mn did not } \\
\text { translate into improve- } \\
\text { ments in child IQ }\end{array}$ & $\begin{array}{l}\text { Maternal IQ and age, } \\
\text { HOME score, child's } \\
\text { school grade, head } \\
\text { circumference and } \\
\text { plasma ferritin }\end{array}$ & High \\
\hline $\begin{array}{l}\text { Zhou } 2019 \\
\text { [39] }\end{array}$ & $6-8$ & $\begin{array}{l}\text { China/ } \\
\text { SMBCS }\end{array}$ & $\begin{array}{l}296(126 / \\
170)\end{array}$ & NA & $\begin{array}{l}\text { Cord blood, } \\
\text { urine }\end{array}$ & WISC & $\begin{array}{l}\text { Urinary Mn: } \uparrow: \\
\text { Performance } \mathrm{IQ} \text { in girls }\end{array}$ & $\begin{array}{l}\text { Child sex, maternal } \\
\text { age, education, } \\
\text { income, inhabitation } \\
\text { area and passive } \\
\text { smoking }\end{array}$ & High \\
\hline
\end{tabular}

\#: 1 year: $n=270$ (131/139); 2 years: $n=430$ (211/219); *:Maternal blood and hair, cord blood, newborns hair, placenta; $\downarrow$ : Negative association; $\uparrow:$ Positive association; NA Not available; NS No significant association. Fe Iron; Hg Mercury; Mn Manganese; Pb Lead. B-Mn Manganese in blood; T-Mn Manganese in teeth. $B M I$ Body mass index; HOME score Home observation for measurement of the environment score; IQ Intelligence Quotient. CHAMACOS The Center for the Health Assessment of Mothers and Children of Salinas study; ELEMENT Early Life Exposures in MExico and NeuroToxicology; ISA Infantes y Salud Ambiental; MOCEH The Mothers and Children's Environmental Health study; SMBCS Sheyang Mini Birth Cohort Study; TBPS The Taiwan Birth Panel Study

(44/55) of included studies was of high quality (see Additional file 2). Except for three studies, all the others described the efforts to address potential sources of bias, such as blinding of exposure status and outcomes assessment, using validated assessment scales and previously trained psychologists.

\section{Manganese in biomarkers and neurodevelopmental outcomes}

In children under 6 years of age, evidence from cohort studies in Table 1 revealed that higher manganese exposure had a negative effect on neurodevelopment [2936], mainly cognitive and motor development. These studies enrolled pregnant women and mainly collected biomarker tissues, such as cord blood, maternal blood and hair, as well as placenta at delivery [29, 30, 32, 3436]. One study sampled maternal hair and blood at intervals 1-3 times during pregnancy [33]. These biomarkers mentioned above were used to indicate prenatal exposure. The other study collected shed teeth from children beginning at age 7 [31], which provides fine scale temporal profiles of $\mathrm{Mn}$ concentrations over the prenatal and early childhood periods. Neurodevelopmental outcomes were assessed by trained psychometricians at follow-up, mainly at 1-2 years of age.

The other two birth cohort studies found an inverted U-shaped association between manganese exposure and cognitive or motor development [27, 28] (see Table 1). Claus Henn et al. (2010) reported that the effect of manganese was apparent for 12-month but diminished for mental development scores at older ages [28], suggesting the possible existence of critical developmental windows. Chung et al. (2015) found a nonlinear dose-response relationship between maternal blood manganese at term 
Table 2 Neurodevelopmental outcomes of manganese exposure measured in biomarkers from cross-sectional studies

\begin{tabular}{|c|c|c|c|c|c|c|c|c|c|}
\hline $\begin{array}{l}\text { Author, } \\
\text { Year }\end{array}$ & $\begin{array}{l}\text { Age } \\
\text { (Years) }\end{array}$ & $\begin{array}{l}\text { Country/ } \\
\text { Study } \\
\text { Name }\end{array}$ & $\begin{array}{l}\text { Number } \\
\text { (Girls/ } \\
\text { Boys) }\end{array}$ & Sources & Biomarkers & $\begin{array}{l}\text { Neurological } \\
\text { Assessments }\end{array}$ & $\begin{array}{l}\text { Associations between } \\
\text { Manganese in } \\
\text { Biomarkers and } \\
\text { Neurodevelopmental } \\
\text { Outcomes }\end{array}$ & $\begin{array}{l}\text { Adjustment for } \\
\text { Covariates }\end{array}$ & $\begin{array}{l}\text { Study } \\
\text { Quality }\end{array}$ \\
\hline $\begin{array}{l}\text { Al-Saleh } \\
2019[40]\end{array}$ & $0.2-1$ & $\begin{array}{l}\text { Saudi } \\
\text { Arabia }\end{array}$ & $\begin{array}{l}206(96 / \\
110)\end{array}$ & NA & $\begin{array}{l}\text { Maternal } \\
\text { blood and } \\
\text { urine, infant } \\
\text { urine, } \\
\text { breast milk }\end{array}$ & DDST-II, PEDS & NS & $\begin{array}{l}\text { Maternal age and } \\
\text { BMI, infant's age, } \\
\text { sex, parity, the } \\
\text { location of primary } \\
\text { health care } \\
\text { centers, maternal } \\
\text { education and z } \\
\text { score weight for } \\
\text { age }\end{array}$ & Medium \\
\hline $\begin{array}{l}\text { Rink } 2014 \\
{[41]}\end{array}$ & $1.1-3.7$ & Uruguay & $\begin{array}{l}60(34 / \\
26)\end{array}$ & NA & Hair & BSID-III & H-Mn: NS & $\begin{array}{l}\text { HOME score, age, } \\
\text { child } \mathrm{Hb} \text {, maternal } \\
\mathrm{IQ}, \mathrm{SES}, \mathrm{Pb} \text {, marital } \\
\text { status, father } \\
\text { education and } \\
\text { tester }\end{array}$ & High \\
\hline $\begin{array}{l}\text { Bauer } 2017 \\
{[42]}\end{array}$ & $10-14$ & Italy/PHIME & $\begin{array}{l}142(79 / \\
63)\end{array}$ & $\begin{array}{l}\text { Fe-Mn alloy } \\
\text { plant }\end{array}$ & Teeth & VRAM & $\begin{array}{l}\text { Both low and high } \\
\text { prenatal Mn } \downarrow \text { : } \\
\text { visuospatial learning } \\
\text { and working memory } \\
\text { among girls only }\end{array}$ & $\begin{array}{l}\text { Sex, age, SES, } \\
\text { videogame use, } \\
\text { lead, trial and } \\
\text { tooth attrition }\end{array}$ & High \\
\hline $\begin{array}{l}\text { Betancourt } \\
2015[43]\end{array}$ & 11 & Ecuador & $\begin{array}{l}93(46 / \\
47)\end{array}$ & $\begin{array}{l}\text { Water } \\
\text { consumption } \\
\text { from the river }\end{array}$ & Hair & PCM & H-Mn: $\downarrow:$ IQ & Mother's literacy & Medium \\
\hline $\begin{array}{l}\text { Bhang } 2013 \\
{[44]}\end{array}$ & $8-11$ & Korea* & $\begin{array}{l}1001 \\
(474 / 527)\end{array}$ & NA & Blood & $\begin{array}{l}\text { WASI, ADS, } \\
\text { CBCL }\end{array}$ & $\begin{array}{l}\text { B-Mn: } \downarrow \text { : academic } \\
\text { performance, such as } \\
\text { thinking, reading, } \\
\text { calculation, lower Mn } \\
\downarrow: \text { attention }\end{array}$ & $\begin{array}{l}\text { Age, sex, region, } \\
\text { children's IQ, } \\
\text { maternal } \\
\text { education and } \\
\text { age, levels of } \\
\text { cotinine and lead }\end{array}$ & High \\
\hline $\begin{array}{l}\text { Bouchard } \\
2007[45]\end{array}$ & $6-15$ & Canada & $\begin{array}{l}46(22 / \\
24)\end{array}$ & Ground water & Hair & $\begin{array}{l}\text { CPRS-R, CTRS- } \\
\mathrm{R}\end{array}$ & $\begin{array}{l}\text { H-Mn: } \downarrow \text { : behaviors } \\
\text { (teacher-reported } \\
\text { hyperactive and } \\
\text { oppositional behaviors) }\end{array}$ & $\begin{array}{l}\text { Child's age, sex } \\
\text { and income }\end{array}$ & High \\
\hline $\begin{array}{l}\text { Bouchard } \\
2011[16]\end{array}$ & $6-13$ & Canada & $\begin{array}{l}362(194 / \\
168)\end{array}$ & Ground water & Hair & WASI & H-Mn: $\downarrow:$ IQ & $\begin{array}{l}\text { Maternal } \\
\text { education and IQ, } \\
\text { income, home } \\
\text { stimulation score, } \\
\text { family structure, } \\
\text { sex and age of } \\
\text { child and IQ } \\
\text { testing session, } \\
\text { source of water } \\
\text { and level of iron in } \\
\text { tap water }\end{array}$ & High \\
\hline $\begin{array}{l}\text { Bouchard } \\
2018[8]\end{array}$ & $6-14$ & Canada & $\begin{array}{l}259(132 / \\
127)\end{array}$ & Ground water & $\begin{array}{l}\text { Hair, saliva, } \\
\text { toe nail }\end{array}$ & WISC-IV & $\begin{array}{l}\text { NS, possible beneficial } \\
\text { effects in boys }\end{array}$ & $\begin{array}{l}\text { Child's age, } \\
\text { maternal IQ and } \\
\text { education, income } \\
\text { and IQ tester }\end{array}$ & High \\
\hline $\begin{array}{l}\text { Carvalho } \\
2014 \text { [22] }\end{array}$ & $7-12$ & Brazil & $\begin{array}{l}70(36 / \\
34)\end{array}$ & $\begin{array}{l}\text { Air emissions } \\
\text { from Fe-Mn } \\
\text { alloy plant }\end{array}$ & Hair & WISC-III & $\begin{array}{l}\text { H-Mn: } \downarrow \text { : estimated IQ, } \\
\text { Block Design and Digit } \\
\text { Span }\end{array}$ & $\begin{array}{l}\text { Maternal } \\
\text { education }\end{array}$ & High \\
\hline $\begin{array}{l}\text { Carvalho } \\
2018[46]\end{array}$ & $7-12$ & Brazil & $\begin{array}{l}70(36 / \\
34)\end{array}$ & $\begin{array}{l}\text { Air emissions } \\
\text { from Fe-Mn } \\
\text { alloy plant }\end{array}$ & Hair & NEPSY ॥ & $\begin{array}{l}\text { H-Mn: } \downarrow \text { : verbal } \\
\text { memory, behaviors } \\
\text { (hyperactivity), not } \\
\text { motor }\end{array}$ & $\begin{array}{l}\text { Age, sex, SES, } \\
\text { mother's } \\
\text { education and } \\
\text { mother's IQ }\end{array}$ & High \\
\hline $\begin{array}{l}\text { Chan } 2015 \\
\text { [47] }\end{array}$ & $11-13$ & $\begin{array}{l}\text { USA } \\
\text { NICHD }\end{array}$ & $\begin{array}{l}266(128 / \\
138)\end{array}$ & NA & Teeth & $\mathrm{DBD}$ & NS & $\begin{array}{l}\text { Child's race, sex, } \\
\text { parental } \\
\text { education, marital } \\
\text { status and SES }\end{array}$ & High \\
\hline
\end{tabular}


Table 2 Neurodevelopmental outcomes of manganese exposure measured in biomarkers from cross-sectional studies (Continued)

\begin{tabular}{|c|c|c|c|c|c|c|c|c|c|}
\hline $\begin{array}{l}\text { Author, } \\
\text { Year }\end{array}$ & $\begin{array}{l}\text { Age } \\
\text { (Years) }\end{array}$ & $\begin{array}{l}\text { Country/ } \\
\text { Study } \\
\text { Name }\end{array}$ & $\begin{array}{l}\text { Number } \\
\text { (Girls/ } \\
\text { Boys) }\end{array}$ & Sources & Biomarkers & $\begin{array}{l}\text { Neurological } \\
\text { Assessments }\end{array}$ & $\begin{array}{l}\text { Associations between } \\
\text { Manganese in } \\
\text { Biomarkers and } \\
\text { Neurodevelopmental } \\
\text { Outcomes }\end{array}$ & $\begin{array}{l}\text { Adjustment for } \\
\text { Covariates }\end{array}$ & $\begin{array}{l}\text { Study } \\
\text { Quality }\end{array}$ \\
\hline $\begin{array}{l}\text { Chiu } 2017 \\
\text { [48] }\end{array}$ & $11-14$ & Italy/PHIME & $\begin{array}{l}194(105 / \\
89)\end{array}$ & $\begin{array}{l}\text { Fe-Mn alloy } \\
\text { plant }\end{array}$ & Teeth & PA, LNMB & $\begin{array}{l}\text { Pretnatal T-Mn: } \uparrow: \text { be- } \\
\text { haviors and motor in } \\
\text { boys, T-Mn: } \downarrow \text { : motor } \\
\text { (tremor): early postna- } \\
\text { tal in girls, later postna- } \\
\text { tal in boys }\end{array}$ & $\begin{array}{l}\text { Children's age and } \\
\text { sex, SES index and } \\
\text { tooth attrition }\end{array}$ & High \\
\hline $\begin{array}{l}\text { do } \\
\text { Nascimento } \\
2015 \text { [49] }\end{array}$ & $6-12$ & Brazil & $\begin{array}{l}69(34 / \\
35)\end{array}$ & $\begin{array}{l}\text { Drinking water } \\
\text { from well } \\
\text { water }\end{array}$ & Hair, blood & RCPM & $\begin{array}{l}\text { H-Mn: } \downarrow \text { : cognitive } \\
\text { function, B-Mn: NS }\end{array}$ & $\begin{array}{l}\text { Age, sex and } \\
\text { parents' education }\end{array}$ & High \\
\hline $\begin{array}{l}\text { Ericson } 2007 \\
{[50]}\end{array}$ & $3-9$ & $\begin{array}{l}\text { USA/ } \\
\text { SECCYD }\end{array}$ & $\begin{array}{l}27(16 / \\
11)\end{array}$ & NA & Teeth & $\mathrm{FTT}, \mathrm{CBCL}$ & $\begin{array}{l}\text { Prenatal T-Mn: } \downarrow \text { : be- } \\
\text { haviors (hyperactivity) }\end{array}$ & $\begin{array}{l}\mathrm{Pb} \text {, mothers' } \\
\text { education, income } \\
\text { and child ethnicity }\end{array}$ & High \\
\hline $\begin{array}{l}\text { Frndak } 2019 \\
\text { [51] }\end{array}$ & $6-8$ & Uruguay & $\begin{array}{l}345(155 / \\
190)\end{array}$ & NA & Hair & CANTAB, W-M & H-Mn: $\uparrow:$ cognition & $\begin{array}{l}\text { Child's age, sex, } \\
\text { Pb, hemoglobin, } \\
\text { HOME score, } \\
\text { crowding, } \\
\text { possessions of } \\
\text { wealth and } \\
\text { mother's } \\
\text { education }\end{array}$ & High \\
\hline $\begin{array}{l}\text { Haynes } \\
2015 \text { [52] }\end{array}$ & $7-9$ & USA/CARES & $\begin{array}{l}404(187 / \\
217)\end{array}$ & $\begin{array}{l}\text { Air-borne Mn } \\
\text { from Fe-Mn } \\
\text { refinery }\end{array}$ & Hair, blood & WISC-IV & $\begin{array}{l}\text { Both low and high Mn: } \\
\downarrow: \text { IQ }\end{array}$ & Parent IQ & Medium \\
\hline $\begin{array}{l}\text { Haynes } \\
2018[7]\end{array}$ & $7-9$ & USA/CARES & $\begin{array}{l}106(65 / \\
41)\end{array}$ & $\begin{array}{l}\text { Air-borne Mn } \\
\text { from Industry }\end{array}$ & Hair, blood & WISC-IV & H-Mn: $\downarrow: I Q, B-M n: N S$ & Parent IQ & Medium \\
\hline $\begin{array}{l}\text { Hernandez- } \\
\text { Bonilla } 2011 \\
\text { [53] }\end{array}$ & $7-11$ & Mexico & $\begin{array}{l}172(84 / \\
88)\end{array}$ & $\begin{array}{l}\text { Air-borne Mn } \\
\text { from Mining }\end{array}$ & Hair, blood & $\mathrm{GP}, \mathrm{FT}, \mathrm{SA}$ & $\begin{array}{l}\text { B-Mn: } \downarrow \text { : motor speed } \\
\text { and coordination, } \mathrm{H}- \\
\text { Mn: NS }\end{array}$ & $\begin{array}{l}\mathrm{Pb}, \mathrm{Hb} \text {, sex, age } \\
\text { and maternal } \\
\text { education }\end{array}$ & High \\
\hline $\begin{array}{l}\text { Hernandez- } \\
\text { Bonilla } 2016 \\
{[54]}\end{array}$ & $7-11$ & Mexico & $\begin{array}{l}267(136 / \\
131)\end{array}$ & $\begin{array}{l}\text { Air-borne Mn } \\
\text { from mining } \\
\text { district }\end{array}$ & Hair & ROCF & $\begin{array}{l}\text { H-Mn: } \downarrow \text { : } \\
\text { visuoperception and } \\
\text { short-term visual } \\
\text { memory }\end{array}$ & $\begin{array}{l}\mathrm{Pb}, \mathrm{Hb} \text {, child's age } \\
\text { and sex, motor } \\
\text { dexterity and } \\
\text { mother's IQ }\end{array}$ & High \\
\hline $\begin{array}{l}\text { Horton } 2018 \\
\text { [55] }\end{array}$ & $8-11$ & $\begin{array}{l}\text { Mexico/ } \\
\text { ELEMENT }\end{array}$ & $\begin{array}{l}133(69 / \\
64)\end{array}$ & $\begin{array}{l}\text { Air pollution } \\
\text { and diets }\end{array}$ & Teeth & BASC-2 & $\begin{array}{l}\text { Prenatal T-Mn: } \uparrow: \text { be- } \\
\text { haviors, postnatal T- } \\
\text { Mn: } \downarrow \text { : behaviors (in- } \\
\text { ternalizing problems) }\end{array}$ & $\begin{array}{l}\text { Maternal } \\
\text { education and } \\
\text { gestational age }\end{array}$ & High \\
\hline $\begin{array}{l}\text { Khan } 2011 \\
{[56]}\end{array}$ & $8-11$ & Bangladesh & $\begin{array}{l}201(100 / \\
101)\end{array}$ & $\begin{array}{l}\text { Drinking water } \\
\text { from well } \\
\text { water }\end{array}$ & Blood & $\mathrm{CBCL}$ & B-Mn: NS & $\begin{array}{l}\text { Arsenic, sex, BMI, } \\
\text { maternal } \\
\text { education and arm } \\
\text { circumference }\end{array}$ & High \\
\hline $\begin{array}{l}\text { Kicinski } \\
2015[57]\end{array}$ & $\begin{array}{l}13.6- \\
17\end{array}$ & Belgium & $\begin{array}{l}606(282 / \\
324)\end{array}$ & $\begin{array}{l}\text { Low-level } \\
\text { metal } \\
\text { exposure from } \\
\text { industrial areas }\end{array}$ & Blood & $\mathrm{FT}, \mathrm{CPT}, \mathrm{DS}$ & B-Mn: NS & $\begin{array}{l}\text { Sex, age, smoking, } \\
\text { passive smoking, } \\
\text { income, } \\
\text { occupation, and } \\
\text { maternal } \\
\text { education }\end{array}$ & High \\
\hline $\begin{array}{l}\text { Kim } 2009 \\
{[58]}\end{array}$ & $8-11$ & Korea & $\begin{array}{l}261(120 / \\
141)\end{array}$ & NA & Blood & WISC & B-Mn: $\downarrow:$ IQ & $\begin{array}{l}\text { Age, sex, parental } \\
\text { education, income, } \\
\text { smoking, birth } \\
\text { weight and } \\
\text { mother's age }\end{array}$ & High \\
\hline $\begin{array}{l}\text { Lucchini } \\
\text { 2012a [59] }\end{array}$ & $11-14$ & Italy & $\begin{array}{l}299(147 / \\
152)\end{array}$ & $\begin{array}{l}\text { Fe-Mn alloy } \\
\text { plant }\end{array}$ & Hair, blood & WISC & NS & $\begin{array}{l}\text { Age, sex, BMI, } \\
\text { family size, SES, } \\
\text { alcohol } \\
\text { consumption, area } \\
\text { of residence, Hb, } \\
\text { ferritin and parity }\end{array}$ & High \\
\hline
\end{tabular}


Table 2 Neurodevelopmental outcomes of manganese exposure measured in biomarkers from cross-sectional studies (Continued)

\begin{tabular}{|c|c|c|c|c|c|c|c|c|c|}
\hline $\begin{array}{l}\text { Author, } \\
\text { Year }\end{array}$ & $\begin{array}{l}\text { Age } \\
\text { (Years) }\end{array}$ & $\begin{array}{l}\text { Country/ } \\
\text { Study } \\
\text { Name }\end{array}$ & $\begin{array}{l}\text { Number } \\
\text { (Girls/ } \\
\text { Boys) }\end{array}$ & Sources & Biomarkers & $\begin{array}{l}\text { Neurological } \\
\text { Assessments }\end{array}$ & $\begin{array}{l}\text { Associations between } \\
\text { Manganese in } \\
\text { Biomarkers and } \\
\text { Neurodevelopmental } \\
\text { Outcomes }\end{array}$ & $\begin{array}{l}\text { Adjustment for } \\
\text { Covariates }\end{array}$ & $\begin{array}{l}\text { Study } \\
\text { Quality }\end{array}$ \\
\hline $\begin{array}{l}\text { Lucchini } \\
\text { 2012b [60] }\end{array}$ & $11-14$ & Italy & $\begin{array}{l}311(153 / \\
158)\end{array}$ & $\begin{array}{l}\text { Fe-Mn alloy } \\
\text { plant }\end{array}$ & $\begin{array}{l}\text { Hair, blood, } \\
\text { urine }\end{array}$ & $\begin{array}{l}\text { FT, PA, DPD, } \\
\text { LNMB }\end{array}$ & $\begin{array}{l}\text { B-Mn and H-Mn: } \downarrow \text { : } \\
\text { motor (tremor), urine, } \\
\text { air, water, diet: NS, soil } \\
\text { Mn: } \downarrow \text { : tremor intensity }\end{array}$ & $\begin{array}{l}\text { Parity, family size, } \\
\text { SES, BMI, maternal } \\
\text { education, alcohol } \\
\text { intake, smoking, } \\
\text { Pb and other } \\
\text { metals in air, soil } \\
\text { and water. }\end{array}$ & High \\
\hline $\begin{array}{l}\text { Lucchini } \\
2019 \text { [61] }\end{array}$ & $6-12$ & Italy & $\begin{array}{l}299(161 / \\
138)\end{array}$ & $\begin{array}{l}\text { Industrial } \\
\text { emission, with } \\
\text { potential } \\
\text { contamination } \\
\text { of } \\
\text { environment }\end{array}$ & Hair & $\begin{array}{l}\text { WISC, CANT } \\
\text { AB }\end{array}$ & $\begin{array}{l}\text { H-Mn: } \downarrow \text { : working } \\
\text { memory }\end{array}$ & $\begin{array}{l}\text { Sex, age, maternal } \\
\text { IQ and cognitive } \\
\text { stimulation } \\
\text { besides the } \\
\text { confounder } \\
\text { distance from the } \\
\text { point source }\end{array}$ & High \\
\hline $\begin{array}{l}\text { Menezes- } \\
\text { Filho } 2011 \\
{[21]}\end{array}$ & $6-12$ & Brazil & $\begin{array}{l}83(39 / \\
44)\end{array}$ & $\begin{array}{l}\text { Fe-Mn alloy } \\
\text { plant }\end{array}$ & Hair, blood & WISC-III & $\begin{array}{l}\text { H-Mn: } \downarrow \text { : cognition, } \\
\text { especially in the verbal } \\
\text { domain, B-Mn: NS }\end{array}$ & $\begin{array}{l}\text { Maternal } \\
\text { education and } \\
\text { nutritional status }\end{array}$ & Medium \\
\hline $\begin{array}{l}\text { Menezes- } \\
\text { Filho } 2014 \\
\text { [62] }\end{array}$ & $7-12$ & Brazil & $\begin{array}{l}70(36 / \\
34)\end{array}$ & $\begin{array}{l}\text { Air-borne Mn } \\
\text { from Fe-Mn } \\
\text { alloy plant }\end{array}$ & Hair & $\mathrm{CBCL}$ & $\begin{array}{l}\text { H-Mn: } \downarrow \text { : behaviors } \\
\text { (externalizing } \\
\text { behaviors), more } \\
\text { pronounced in girls }\end{array}$ & $\begin{array}{l}\text { Age, sex and } \\
\text { maternal IQ }\end{array}$ & High \\
\hline $\begin{array}{l}\text { Nascimento } \\
2016 \text { [63] }\end{array}$ & $6-12$ & Brazil & $\begin{array}{l}63(31 / \\
32)\end{array}$ & $\begin{array}{l}\text { Potential } \\
\text { contamination } \\
\text { from pesticide }\end{array}$ & Hair, blood & NEUPSILIN-Inf & $\begin{array}{l}\text { B-Mn: } \downarrow \text { : visual } \\
\text { attention, visual } \\
\text { perception and } \\
\text { phonological } \\
\text { awareness, H-Mn: } \downarrow \text { : } \\
\text { working memory }\end{array}$ & $\begin{array}{l}\text { IQ, age, sex and } \\
\text { parents' education }\end{array}$ & High \\
\hline $\begin{array}{l}\text { Oulhote } \\
2014 \text { [15] }\end{array}$ & $6-13$ & Canada & $\begin{array}{l}375(200 / \\
175)\end{array}$ & $\begin{array}{l}\text { Drinking water } \\
\text { from ground } \\
\text { water }\end{array}$ & Hair & $\begin{array}{l}\text { WASI, CPT-II, } \\
\text { FT, SA }\end{array}$ & $\begin{array}{l}\text { H-Mn: } \downarrow \text { : memory, } \\
\text { attention, not } \\
\text { hyperactivity, motor: a } \\
\text { nonlinear association, } \\
\uparrow: 0.3-0.8 \mu \mathrm{g} / \mathrm{g}, \downarrow:> \\
10 \mu \mathrm{g} / \mathrm{g}, \mathrm{but} \text { there } \\
\text { were very few } \\
\text { observations with such } \\
\text { high levels }\end{array}$ & $\begin{array}{l}\text { Child's sex, age, } \\
\text { maternal } \\
\text { education and IQ, } \\
\text { income, maternal } \\
\text { depressive } \\
\text { symptoms and tap } \\
\text { water lead } \\
\text { concentrations. }\end{array}$ & High \\
\hline $\begin{array}{l}\text { Parvez } 2011 \\
\text { [64] }\end{array}$ & $8-11$ & Bangladesh & $\begin{array}{l}304(153 / \\
151)\end{array}$ & $\begin{array}{l}\text { Drinking water } \\
\text { from well } \\
\text { water }\end{array}$ & Blood & BOT-2 & B-Mn: NS & $\begin{array}{l}\text { Sex, school } \\
\text { attendance, head } \\
\text { circumference, } \\
\text { mother's } \\
\text { intelligence, } \\
\text { ferritin, selenium } \\
\text { and } \mathrm{Pb}\end{array}$ & High \\
\hline $\begin{array}{l}\text { Riojas- } \\
\text { Rodríguez } \\
2010[6]\end{array}$ & $7-11$ & Mexico & $\begin{array}{l}172(99 / \\
73)\end{array}$ & $\begin{array}{l}\text { Air-borne Mn } \\
\text { from mining } \\
\text { district }\end{array}$ & Hair, blood & WISC & H-Mn: $\downarrow$ : IQ, B-Mn: NS & $\begin{array}{l}\mathrm{Pb} \text {, age, sex, } \\
\text { nutritional status, } \\
\text { maternal } \\
\text { education and IQ }\end{array}$ & High \\
\hline $\begin{array}{l}\text { Rugless } \\
2014 \text { [65] }\end{array}$ & $7-9$ & USA/CARES & $\begin{array}{l}55(35 / \\
20)\end{array}$ & $\begin{array}{l}\text { Air emissions } \\
\text { from Fe-Mn } \\
\text { refinery }\end{array}$ & Hair, blood & APS & $\begin{array}{l}\text { H-Mn and B-Mn: } \downarrow \text { : } \\
\text { postural balance }\end{array}$ & $\begin{array}{l}\text { Sex, height/weight } \\
\text { ratio, parent } \mathrm{IQ} \text {, } \\
\text { education, } \mathrm{Pb} \text { and } \\
\text { age }\end{array}$ & Medium \\
\hline $\begin{array}{l}\text { Torrente } \\
2005[66]\end{array}$ & $12-14$ & Spain & $\begin{array}{l}100(61 / \\
39)\end{array}$ & $\begin{array}{l}\text { Industrial } \\
\text { emission }\end{array}$ & Hair & AMP & H-Mn: NS & SES and age & Low \\
\hline $\begin{array}{l}\text { Torres- } \\
\text { Agustin } \\
2013[67]\end{array}$ & $7-11$ & Mexico & $\begin{array}{l}174(86 / \\
88)\end{array}$ & $\begin{array}{l}\text { Air-borne Mn } \\
\text { from mining } \\
\text { district }\end{array}$ & Hair, blood & CAVLT & $\begin{array}{l}\text { H-Mn: } \downarrow \text { : long-term } \\
\text { memory and learning, } \\
\text { B-Mn: NS }\end{array}$ & $\begin{array}{l}\text { Child's sex, } \mathrm{Pb} \text {, } \\
\text { age, } \mathrm{Hb} \text { and } \\
\text { maternal } \\
\text { education }\end{array}$ & High \\
\hline Wasserman & 10 & Bangladesh & $142(72 /$ & Drinking water & Blood & WISC-III & B-Mn: NS & Maternal & High \\
\hline
\end{tabular}


Table 2 Neurodevelopmental outcomes of manganese exposure measured in biomarkers from cross-sectional studies (Continued)

\begin{tabular}{|c|c|c|c|c|c|c|c|c|c|}
\hline $\begin{array}{l}\text { Author, } \\
\text { Year }\end{array}$ & $\begin{array}{l}\text { Age } \\
\text { (Years) }\end{array}$ & $\begin{array}{l}\text { Country/ } \\
\text { Study } \\
\text { Name }\end{array}$ & $\begin{array}{l}\text { Number } \\
\text { (Girls/ } \\
\text { Boys) }\end{array}$ & Sources & Biomarkers & $\begin{array}{l}\text { Neurological } \\
\text { Assessments }\end{array}$ & $\begin{array}{l}\text { Associations between } \\
\text { Manganese in } \\
\text { Biomarkers and } \\
\text { Neurodevelopmental } \\
\text { Outcomes }\end{array}$ & $\begin{array}{l}\text { Adjustment for } \\
\text { Covariates }\end{array}$ & $\begin{array}{l}\text { Study } \\
\text { Quality }\end{array}$ \\
\hline 2006 [9] & & & 70) & $\begin{array}{l}\text { from well } \\
\text { water }\end{array}$ & & & & $\begin{array}{l}\text { education and } I Q \text {, } \\
\text { house type, family } \\
\text { ownership of a } \\
\text { television, child } \\
\text { height and head } \\
\text { circumference }\end{array}$ & \\
\hline $\begin{array}{l}\text { Wright } 2006 \\
\text { [68] }\end{array}$ & $11-13$ & USA & $\begin{array}{l}31(16 / \\
15)\end{array}$ & Mining waste & Hair & WASI & $\begin{array}{l}\text { H-Mn: } \downarrow: \text { Full Scale IQ, } \\
\text { Verbal IQ }\end{array}$ & $\begin{array}{l}\text { Sex and maternal } \\
\text { education }\end{array}$ & Low \\
\hline
\end{tabular}

*: Effects of pollution on neurobehavioral development, and future policies to protect our children; $\downarrow$ : Negative association; $\uparrow:$ Positive association; $N A$ Not available; NS No significant association. Fe-Mn Ferro-manganese; Hb Hemoglobin; Mn Manganese; $P b$ Lead. $B-M n$ Manganese in blood; $H-M n$ Manganese in hair; $T$ $M n$ Manganese in teeth. BMI Body mass index; HOME score Home observation for measurement of the environment score; IQ Intelligence Quotient; SES Socioeconomic status. CARES Communities Actively Researching Exposure Study; ELEMENT Early Life Exposures in MExico and NeuroToxicology; NICHD National Institute of Child Health and Human Development; PHIME Public Health Impact of Manganese Exposure in susceptible populations, SECCYD Study of Early Child Care and Youth Development

and 6-month psychomotor development scores, with a peak point approximately $24-28 \mu \mathrm{g} / \mathrm{L}$, suggesting adverse neurodevelopmental effects of both low $(<20.0 \mu \mathrm{g} /$ L) and high $(\geq 30.0 \mu \mathrm{g} / \mathrm{L})$ maternal blood manganese concentrations [27].

The results from cohort studies concerning children over 6 years old were intriguing. Two follow-up studies in Bangladesh and Canada were conducted to evaluate whether changes in drinking water manganese exposure were associated with changes in child intellectual outcomes. In Bangladesh, Wasserman et al. (2016) found that during 2 years of follow-up, the reduction in exposure (indicated by manganese in blood, B-Mn) was not, for the most part, translated into improvements in child IQ. In this cohort, baseline B-Mn was negatively associated with working memory after covariate adjustment [19]. In Quebec (Canada), the result revealed that, for children whose Mn concentrations in their water supply increased between baseline and follow-up, their Performance IQ scores decreased significantly. On the other hand, at follow-up, higher manganese in drinking water was associated with lower Performance IQ in girls, whereas the opposite was observed in boys. Similar trends were observed in hair [20]. Although the results of cohort studies need to be verified, they also suggest the importance of preventing such exposures.

Inconsistent conclusions were drawn from three cohort studies, one measured manganese in cord blood and spot urine [39], the other two sampled dentine of incisors $[37,38]$ (see Table 1). The birth cohort study in China reported that urinary Mn concentrations, but not cord blood manganese, were positively associated with Performance IQ of school-age children, especially in girls [39]. Mora et al. (2015) found that higher prenatal and early postnatal manganese in teeth (T-Mn) were adversely associated with behavioral outcomes, namely internalizing, externalizing and hyperactivity problems, in children at 7 and 10.5 years. In the sex stratified models, Mora et al.(2015) found that higher prenatal and postnatal T-Mn were associated with better memory abilities at ages 9 and 10.5, and better cognitive and motor outcomes at ages 7 and 10.5 years, among boys only [38]. On the other hand, Claus Henn et al. (2018) found that higher postnatal T-Mn was negatively associated with both Wide Range Assessment of Visual Motor Abilities (WRAVMA) total and visual spatial subtest scores, among boys only [37]. Mn interactions with lead $(\mathrm{Pb})$ were also examined. Mora et al. (2015) reported that higher prenatal T-Mn was associated with poorer visuospatial memory outcomes at 9 years and worse cognitive scores at 7 and 10.5 years in children with higher prenatal blood lead concentrations $(\geq 0.8 \mu \mathrm{g} / \mathrm{dL})$ [38]. And Claus Henn et al. (2018) found that tooth Mn was positively associated with visual spatial and total WRAV MA scores in the second trimester, among children with lower (< median) tooth $\mathrm{Pb}$ concentrations, while no significant $\mathrm{Mn}$ association was observed at high $\mathrm{Pb}$ concentrations [37]. These inconsistent findings may be due to differences in biomarkers (blood and urine vs. teeth) or sources of Mn exposure (Mn-containing fungicides vs. dietary and airborne sources).

Although most cohort studies found adverse association between manganese exposure and neurodevelopment, $\mathrm{Mn}$ interactions with sex and other metals, such as $\mathrm{Pb}$, were gaining attention. Among these studies, only six studies described the sources of manganese exposure during pregancy, such as mining, mancozeb and drinking water, the concentration of manganese was only measured in drinking water in two studies $[19,20]$. And Dion et al. (2018) reported that Mn in hair (H-Mn) correlated with W-Mn at follow-up $(r, 0.48 ; p<0.001)$ and with time-averaged W-Mn $(r, 0.43 ; p<0.001)$ [20]. 
Table 3 Neurodevelopmental outcomes of manganese exposure measured in drinking water

\begin{tabular}{|c|c|c|c|c|c|c|c|c|}
\hline $\begin{array}{l}\text { Author, } \\
\text { Year }\end{array}$ & $\begin{array}{l}\text { Age } \\
\text { (Years) }\end{array}$ & $\begin{array}{l}\text { Country/ } \\
\text { Study } \\
\text { Name }\end{array}$ & $\begin{array}{l}\text { Number } \\
\text { (Girls/ } \\
\text { Boys) }\end{array}$ & Sources & $\begin{array}{l}\text { Neurological } \\
\text { Assessments }\end{array}$ & $\begin{array}{l}\text { Associations between } \\
\text { Manganese in } \\
\text { Drinking Water and } \\
\text { Neurodevelopmental } \\
\text { Outcomes }\end{array}$ & Adjustment for Covariates & $\begin{array}{l}\text { Study } \\
\text { Quality }\end{array}$ \\
\hline \multicolumn{9}{|c|}{ Neurodevelopmental outcomes from cohort studies } \\
\hline $\begin{array}{l}\text { Dion } 2018 \\
\text { [20] }\end{array}$ & $\begin{array}{l}10.5- \\
18\end{array}$ & Canada & $\begin{array}{l}287(151 / \\
136)\end{array}$ & Ground water & WASI & $\begin{array}{l}\text { W-Mn and time- } \\
\text { averaged W-Mn: } \downarrow: \text { IQ } \\
\text { among girls }\end{array}$ & $\begin{array}{l}\text { Maternal IQ, education and } \\
\text { income }\end{array}$ & High \\
\hline $\begin{array}{l}\text { Rahman } \\
2017 \text { [69] }\end{array}$ & 10 & Bangladesh & $\begin{array}{l}1265 \\
(609 / 656)\end{array}$ & Well water & WISC-IV, SDQ & $\begin{array}{l}\text { Prenatal W-Mn: } \uparrow: \text { cog- } \\
\text { nition in girls, W-Mn: } \downarrow \text { : } \\
\text { behavior }\end{array}$ & $\begin{array}{l}\text { Maternal IQ, SES, child age, sex, } \\
\text { education, height for age, } \mathrm{Hb} \text {, } \\
\text { school type, HOME, tester, } \\
\text { number of siblings and arsenic }\end{array}$ & High \\
\hline $\begin{array}{l}\text { Rodrigues } \\
2016 \text { [70] }\end{array}$ & $1.6-3.3$ & Bangladesh & $\begin{array}{l}525(264 / \\
261)\end{array}$ & $\begin{array}{l}\text { Drinking water } \\
\text { from well } \\
\text { water }\end{array}$ & BSID-III & $\begin{array}{l}\text { W-Mn: an inverse-U re- } \\
\text { lationship with fine } \\
\text { motor function, cogni- } \\
\text { tion: NS }\end{array}$ & $\begin{array}{l}\text { Maternal age, maternal } \\
\text { education, passive smoking, } \\
\text { child's sex, HOME score, maternal } \\
\text { IQ and child's hematocrit levels }\end{array}$ & High \\
\hline \multicolumn{9}{|c|}{ Neurodevelopmental outcomes from cross-sectional studies } \\
\hline $\begin{array}{l}\text { Bouchard } \\
2011[16]\end{array}$ & $6-13$ & Canada & $\begin{array}{l}362(194 / \\
168)\end{array}$ & Ground water & WASI & $\begin{array}{l}\text { W-Mn and estimated } \\
\text { Mn intake from water } \\
\text { consumption: } \downarrow \text { : IQ, } \\
\text { estimated Mn intake } \\
\text { from dietary: NS }\end{array}$ & $\begin{array}{l}\text { Maternal education and IQ, } \\
\text { income, home stimulation score, } \\
\text { family structure, sex and age of } \\
\text { child, IQ testing session, source } \\
\text { of water and level of iron in tap } \\
\text { water }\end{array}$ & High \\
\hline $\begin{array}{l}\text { Bouchard } \\
2018[8]\end{array}$ & $6-14$ & Canada & $\begin{array}{l}259(132 / \\
127)\end{array}$ & $\begin{array}{l}\text { Drinking water } \\
\text { from ground } \\
\text { water }\end{array}$ & WISC-IV & $\begin{array}{l}\text { NS, possible beneficial } \\
\text { effects in boys }\end{array}$ & $\begin{array}{l}\text { Child's age, maternal IQ and } \\
\text { education, income and IQ tester }\end{array}$ & High \\
\hline $\begin{array}{l}\text { do } \\
\text { Nascimento } \\
2015[49]\end{array}$ & $6-12$ & Brazil & $\begin{array}{l}69(34 / \\
35)\end{array}$ & $\begin{array}{l}\text { Drinking water } \\
\text { from well } \\
\text { water }\end{array}$ & RCPM & $\begin{array}{l}\text { W-Mn: } \downarrow \text { : cognitive } \\
\text { function }\end{array}$ & Age, sex and parents' education & High \\
\hline $\begin{array}{l}\text { Khan } 2011 \\
{[56]}\end{array}$ & $8-11$ & Bangladesh & $\begin{array}{l}201(100 / \\
101)\end{array}$ & $\begin{array}{l}\text { Drinking water } \\
\text { from well } \\
\text { water }\end{array}$ & $\mathrm{CBCL}$ & $\begin{array}{l}\text { W-Mn: } \downarrow \text { : behaviors } \\
\text { (classroom behavioral } \\
\text { problems) }\end{array}$ & $\begin{array}{l}\text { Arsenic, sex, BMI, maternal } \\
\text { education and arm } \\
\text { circumference }\end{array}$ & High \\
\hline $\begin{array}{l}\text { Khan } 2012 \\
\text { [71] }\end{array}$ & $8-11$ & Bangladesh & $\begin{array}{l}840(444 / \\
396)\end{array}$ & Well water & AARES & $\begin{array}{l}W-M n>400 \mu \mathrm{g} / \mathrm{L}: \downarrow: \\
\text { mathematics test }\end{array}$ & $\begin{array}{l}\text { School-grade, parental education } \\
\text { and head circumference and } \\
\text { controlling for within-teacher } \\
\text { correlations in rating the children }\end{array}$ & High \\
\hline $\begin{array}{l}\text { Nascimento } \\
2016[63]\end{array}$ & $6-12$ & Brazil & $\begin{array}{l}63(31 / \\
32)\end{array}$ & $\begin{array}{l}\text { Potential } \\
\text { contamination } \\
\text { from pesticide }\end{array}$ & NEUPSILIN-Inf & $\begin{array}{l}\text { W-Mn: } \downarrow \text { : written } \\
\text { language and } \\
\text { executive functions }\end{array}$ & $\begin{array}{l}I Q \text {, age, sex and parents' } \\
\text { education }\end{array}$ & High \\
\hline $\begin{array}{l}\text { Oulhote } \\
2014 \text { [15] }\end{array}$ & $6-13$ & Canada & $\begin{array}{l}375(200 / \\
175)\end{array}$ & $\begin{array}{l}\text { Drinking water } \\
\text { from ground } \\
\text { water }\end{array}$ & $\begin{array}{l}\text { WASI, CPT-II, } \\
\text { FT, SA }\end{array}$ & $\begin{array}{l}\text { W-Mn: } \downarrow \text { : memory, Mn } \\
\text { intake from water: } \downarrow \text { : } \\
\text { motor function }\end{array}$ & $\begin{array}{l}\text { Child's sex, age, maternal } \\
\text { education and IQ, income, } \\
\text { maternal depressive symptoms } \\
\text { and tap water lead }\end{array}$ & High \\
\hline $\begin{array}{l}\text { Wasserman } \\
2006[9]\end{array}$ & 10 & Bangladesh & $\begin{array}{l}142(72 / \\
70)\end{array}$ & $\begin{array}{l}\text { Drinking water } \\
\text { from well } \\
\text { water }\end{array}$ & WISC-III & W-Mn: $\downarrow:$ IQ & $\begin{array}{l}\text { Maternal education and IQ, } \\
\text { house type, family ownership of } \\
\text { a television, child height and } \\
\text { head circumference }\end{array}$ & High \\
\hline
\end{tabular}

$\downarrow$ : Negative association; $\uparrow$ : Positive association; NS No significant association. Hb Hemoglobin; Mn Manganese. W-Mn Manganese in drinking water. BMI Body mass index; HOME score Home observation for measurement of the environment score; IQ Intelligence Quotient; SES Socioeconomic status

Two out of the 37 cross-sectional studies investigated associations between Mn exposure and developmental scores in infants. Postnatal manganese exposure were measured in breast milk, blood, urine and hair, no significant association was observed [40, 41], with significantly negative association in the unadjusted model [41] (see Table 2).
There were 35 studies concerning children over 6 years old, and these also measured manganese in related biomarkers, such as hair $(n=24)[6-8,15,16,21,22,43,45$, $46,49,51-54,59-63,65-68]$, blood $(n=17)[6,7,9,21$, $44,49,52,53,56-60,63-65,67]$, teeth $(n=5)[42,47,48$, $50,55]$, saliva, toe nail [8] and urine [60]. One study could be included when using more than one biomarker, as was 
the case in New Brunswick (Canada), which measured manganese in hair, saliva and toe nail [8].

A central result was that elevated $\mathrm{H}-\mathrm{Mn}$ was associated with poorer cognitive and behavioral performance in most studies $(n=17)$, in terms of IQ $[6,7,16,21,22$, $43,49,52,68]$, working memory [61, 63], verbal memory [46], visuoperception and short-term visual memory [54], long-term memory and learning abilities [67], memory and attention [15], hyperactivity behaviors [45, 46], oppositional behaviors [45] and externalizing behavioral problems [62] (see Table 2). Among them, Oulhote et al. (2014) found that there was no significant association between manganese exposure and hyperactivity [15]. In this case, a large percent (13/17) of the studies reported that the mean of manganese in hair exceeded $0.55 \mu \mathrm{g} / \mathrm{g}$, which was similar to the mean concentration from control groups [6, 54]. Haynes et al. (2015) also found that compared with the middle two quartiles, the lowest quartiles of $\mathrm{H}-\mathrm{Mn}(<0.21 \mu \mathrm{g} / \mathrm{g})$ was associated with significantly lower mean perceptual reasoning scores [52]. Similarly, one cross-sectional study revealed a positive association between $\mathrm{H}-\mathrm{Mn}$ and cognitive function in children aged at 6-8 years, with a low median concentration of $\mathrm{Mn}$ in hair $(0.82 \mathrm{ng} / \mathrm{g})$ [51]. No significant associations were found in three studies in terms of cognitive functions $[8,59,66]$ and behavioral performance [59], with the average of manganese in hair ranged from $0.17 \mu \mathrm{g} / \mathrm{g}$ to $0.3 \mu \mathrm{g} / \mathrm{g}$. For motor function, two studies showed that elevated $\mathrm{H}-\mathrm{Mn}$ was associated with tremor intensity [60] and poor postural balance [65], while two articles found no association between $\mathrm{H}-\mathrm{Mn}$ and motor function [46, 53]. In another publication, Oulhote et al. (2014) found a nonlinear association between $\mathrm{H}-\mathrm{Mn}$ and motor function, with a slight increase at concentrations between 0.3 and $0.8 \mu \mathrm{g} / \mathrm{g}$, and an apparent decrease in scores at $\mathrm{H}-\mathrm{Mn}>10 \mu \mathrm{g} / \mathrm{g}$, although there were very few observations with such high concentrations [15]. This inconsistency may possibly due to the different levels of manganese exposure and the sensitivity of scales, as the average concentration of $\mathrm{H}-\mathrm{Mn}$ ranged widely from $0.16 \mu \mathrm{g} / \mathrm{g}$ to $14.6 \mu \mathrm{g} / \mathrm{g}[15,46,53,60$, 65]. Carvalho et al. (2018) reported poorer cognition and behavior, while no effect on motor in the same exposure population [46] (see Table 2).

In contrast to hair, most $(n=9)$ reports in Table 2 indicated that B-Mn was non-significantly associated with cognitive and behavioral development $[6,7,9,21,49,56$, $57,59,67]$. However, two studies did show that elevated B-Mn was associated with poorer cognitive development, when using IQ [58], visual attention, visual perception and phonological awareness [63] as outcome measures. Two publications suggested that both low and high BMn were negatively associated with cognitive and behavioral development $[44,52]$. In relation to motor development, three studies showed that elevated B-Mn was associated with impairment of motor functions, namely tremor intensity [60], postural balance [65], coordination and motor speed [53]. However, one study indicated no significant association [64] (see Table 2). Among these reports, the mean concentration of manganese in blood was mainly around $10 \mu \mathrm{g} / \mathrm{L}$, suggesting the relatively homeostatic regulation of blood manganese.

Five publications used teeth as a biomarker [42, 47, 48, $50,55]$ (see Table 2). One study found an inverted Ushaped association between prenatal $\mathrm{Mn}$ and visuospatial ability in girls, no significant associations were found in postnatal Mn [42]. Ericson et al. (2007) found that higher $\mathrm{Mn}$ in teeth was adversely associated with behavioral outcomes [50]. Horton et al.(2018) revealed that prenatal $\mathrm{Mn}$ exposure appeared to be protective against behavioral outcomes, yet postnatal Mn appeared as a risk factor for behavioral outcomes [55]. Two of which indicated that there were no significant associations between $\mathrm{Mn}$ in deciduous teeth and behavioral [47] and motor development [48]. These results suggested that $\mathrm{Mn}$ associations were partly driven by exposure timing and modified by sex. Three studies also found that tooth $\mathrm{Mn}$ concentrations were higher in the prenatal than postnatal period [42, 48, 55], indicating a greater demand for manganese in the prenatal period. No significant associations were observed between neurodevelopmental outcomes and $\mathrm{Mn}$ in saliva, toe nail [8], and urine [60] (see Table 2).

\section{Manganese in drinking water and neurodevelopmental outcomes}

Evidience from cohort studies indicated that elevated W-Mn was associated with lower IQ scores in girls [20] and the increased risk of children's behavioral problems at 10 years of age [69] (see Table 3). While Rodrigues et al. (2016) also found an inverted U-shaped association between W-Mn and motor development with an inflection point around $400 \mu \mathrm{g} / \mathrm{L}$ [70].

Most $(n=7)$ cross-sectional studies found that higher W-Mn was associated with poorer cognitive and behavioral function, such as IQ $[9,16,49]$, memory [15], written language [63], mathematics scores [71] and the risk of behavioral problems [56]. The mean concentrations of W-Mn were shown to range from 795 to $1387.9 \mu \mathrm{g} / \mathrm{L}$ in three studies conducted in Araihazar, a rural area of Bangladesh $[9,56,71]$, which were much higher than W-Mn in Canada, with its arithmetic mean of $98 \mu \mathrm{g} / \mathrm{L}$ reported in two studies $[15,16]$. The W-Mn in two studies conducted in Brazil was much lower, with the mean of W-Mn around $20 \mu \mathrm{g} / \mathrm{L}$ in the rural group [49, 63]. By contrast, two reports found no clear association between W-Mn and childhood IQ [8] and behavioral function [15] (see Table 3). Both studies were conducted in 
Canada $[8,15]$, one described a situation where W-Mn was low, with approximately half of children's home tap water with a manganese concentration less than $5 \mu \mathrm{g} / \mathrm{L}$ [8]. For motor function, the association with W-Mn was significant, with a threshold indicating that scores decreased more steeply at concentrations above $180 \mu \mathrm{g} / \mathrm{L}$, and this research also found that manganese intake from water was negatively associated with motor function [15]. Of note, most studies also measured manganese in hair or blood, the conclusions were mainly consistent with $\mathrm{H}-\mathrm{Mn}[15,16,49,63]$, whereas the inconsistency was observed in blood $[9,56]$.

\section{Pooled effect estimates for IQ scores}

The details for our meta-analysis were extracted to include three manganese exposure metrics: hair, blood and drinking water, as shown in Additional file 3. Among these studies, seven researches had a cross-sectional design, two cohort studies were treated as cross-sectional studies by using the associations between Mn exposure and concurrent IQ scores at baseline examinations [19] or follow-up examinations [20].

Figure 2 clearly shows that a 10 -fold increase in hair manganese is associated with a decrease of 2.51 points
(95\% CI, $\left.-4.58,-0.45 ; I^{2}=59.8 \%\right)$ in Full Scale IQ of children aged 6-18 years. Of note, this inverse relationship remained significant when we conducted a sensitivity analysis in which one study was removed at a time (see Additional file 4). The pooled results with respect to Performance IQ were not extremely robust and should be investigated further. Heterogeneity was $59.8 \%$ for Full Scale IQ, as a "leave-one-out" analysis revealed that there still existed some heterogeneities. Next, we performed a subgroup analysis based on the source of exposure, revealing a significantly inverse association between $\mathrm{H}-\mathrm{Mn}$ and IQ scores from airborne manganese exposure $[6,21,22]$, but not from waterborne $[8,16,20]$ or mining waste manganese exposure [68]. The pooled $\beta$ for the 10-fold increase in hair manganese from airborne manganese exposure was associated with a decrease of 7.62 points $\left(95 \% \mathrm{CI},-11.51,-3.73 ; I^{2}=0 \%\right)$ for Full Scale IQ. For Performance IQ, the decrease would be 2.60 points $\left(95 \% \mathrm{CI},-3.94,-1.25 ; I^{2}=0 \%\right)$, and 4.56 points decrease $\left(95 \% \mathrm{CI},-8.33,-0.79 ; I^{2}=45.5 \%\right)$ for Verbal IQ. Unexpectedly, the concentrations of $\mathrm{H}-\mathrm{Mn}$ from airborne manganese exposure were much higher than the others. Therefore, we concluded that both the source of manganese exposure and the concentrations of

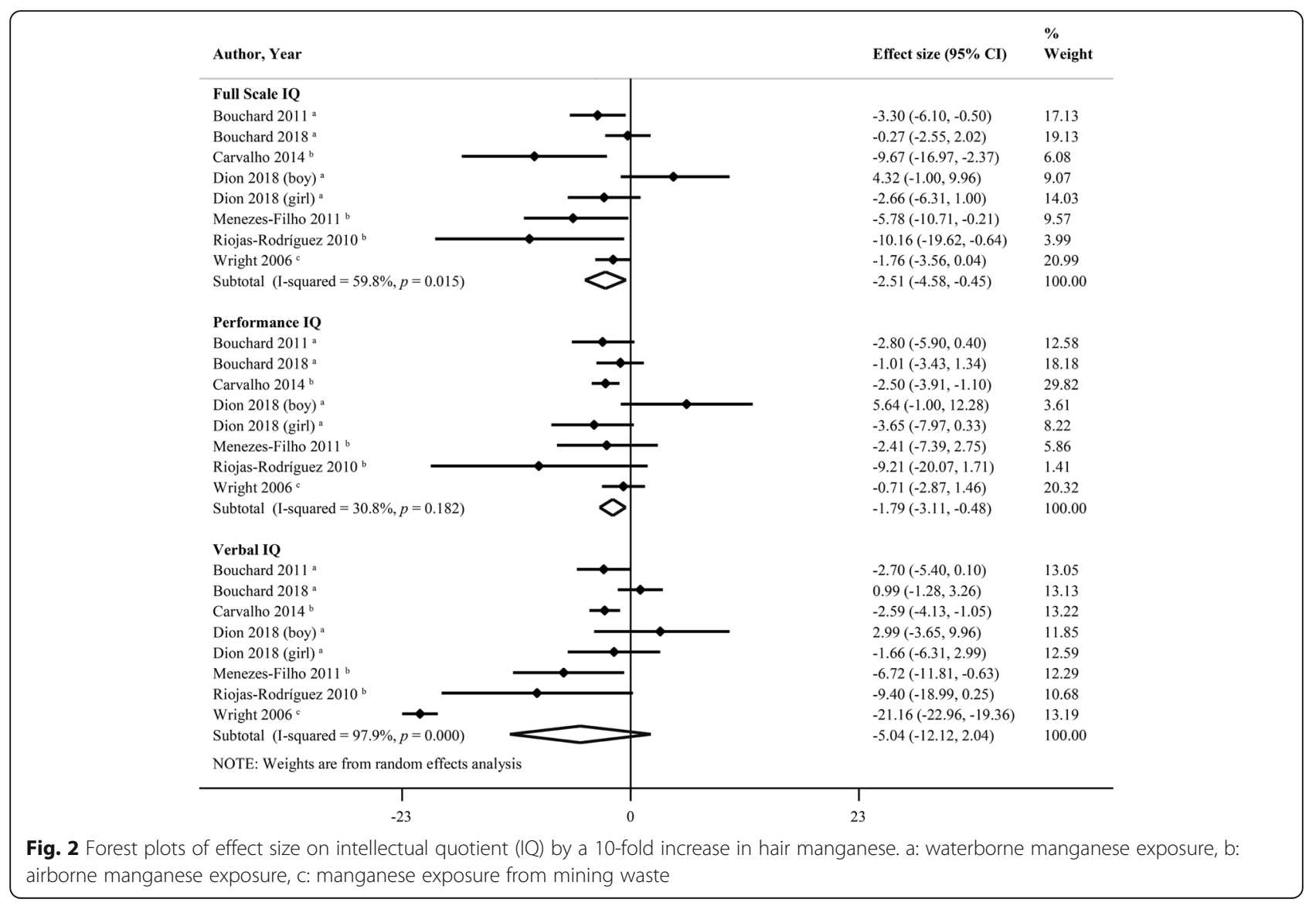


H-Mn likely account, at least in part, for this relatively high heterogeneity. The results from Begg's and Egger's tests did not suggest the existence of publication bias.

The meta-analysis in drinking water and blood revealed no significant effects (Additional files 5, 6). Of significance, among the reports that used both hair and blood as biomarkers, a large percent (7/11) of which indicated that H-Mn, but not B-Mn, was negatively associated with cognitive development $[6,7,21,49,52,59,67]$ (Table 2).

\section{Different biomarkers in $\mathrm{Mn}$ exposure and neurodevelopment}

In the elder group, most studies used hair and blood as biomarkers. While teeth was also used as a biomarker in some publications with inconsistent $\mathrm{Mn}$ associations. Some researches also measured manganese in environmental samples, such as drinkable water, soil and particles. The correlations between manganese in drinking water and biomarker (hair or blood) or different biomarkers were analyzed in nine studies, as shown in Additional file 7. Among these publications, six studies used Spearman's rank correlation $[7,9,49,56,63,67]$, as the distributions of manganese concentrations in biomarkers and drinking water were considerably skewed. Three studies analyzed the correlation using Pearson correlation tests, among these studies, the concentrations of manganese in indicators were transformed in order to make distributions more symmetrical for Pearson correlation tests [20, 21, 52].

The preliminary meta-analysis was conducted to gain a pooled result of correlations between different manganese indicators (see Additional file 7). The correlation between $\mathrm{H}-\mathrm{Mn}$ and $\mathrm{W}-\mathrm{Mn}$ indicated that they did have significance, and the pooled correlation coefficient $r$ was 0.48 ( $95 \% \mathrm{CI}, 0.40,0.55)$. By contrast, the summary correlation between B-Mn and W-Mn, even B-Mn and $\mathrm{H}$ $\mathrm{Mn}$ indicated that there had no significance. Although different analytical methods were applied in three studies that analyzed the correlation between $\mathrm{H}-\mathrm{Mn}$ and W$\mathrm{Mn}$, the conclusion was consistent $[20,49,63]$.

Wasserman et al. (2011) found that blood did not vary predictably across the low and high W-Mn groups, suggesting that blood may not be a good reflection of drinking water $\mathrm{Mn}$ exposure [72]. From the airborne manganese exposure, Torres-Agustin et al. (2013) observed a statistically significant difference between the two groups in the median blood Mn concentrations of 8.0 and $9.5 \mu \mathrm{g} / \mathrm{L}$ for non-exposed and exposed children, respectively. Meanwhile, hair Mn concentrations in exposed children were, on average, 20 times higher (median 12.6 and mean $14.2 \mu \mathrm{g} / \mathrm{g}$ ) than the nonexposed group (median 0.6 and mean $0.73 \mu \mathrm{g} / \mathrm{g}$ ) [67]. These results indicate that hair is more sensitive than blood to reflect environmental manganese exposure.

In infants, one report found that maternal and cord blood manganese concentrations were correlated, though in a nonlinear manner. Here, the median manganese in cord blood was nearly twice the median concentration in maternal blood, unexpectedly, the inverse associations were found between manganese in maternal blood, but not cord blood, and early childhood mental and psychomotor development scores [29]. The other biomarkers (i.e. maternal and infant hair and placenta) used to reflect prenatal Mn exposure did not show the correlation.

\section{Sex specific exposure-response relationships}

Evidence from eight cohort studies yielded inconsistent conclusions as to sexual effects (see Table 4). Three of four studies reported a statistically significant sex interaction $(p<0.05)$, and concluded that girls were more susceptible to manganese exposure than boys in terms of cognition and motor [20,31,33]. While Takser et al. (2003) found that in children at 3 years, the hand skill score was negatively associated with cord blood $\mathrm{Mn}$ in boys $(p=0.002)$, but not in girls [34]. Two cohort studies found a positive non-statistically significant association between manganese exposure and cognitive development in girls [69] and cognitive and motor development in boys [38]. Sex interaction $p$-values in the remaining two reports were not available. Positive association between urinary Mn concentrations and Performance IQ of children was observed, especially in girls [39]. And Claus Henn et al. (2018) found significantly negative associations between $\mathrm{T}-\mathrm{Mn}$ and visual spatial score, among boys only [37].

Seven cross-sectional studies consistently concluded that girls were more susceptible to manganese exposure than boys with respect to cognition and behavior $[6,8$, $16,42,54,62,67]$, with non-statistically significant association in three reports $[16,42,54]$ and interaction $p$ values not available in four studies $[6,8,62,67]$. Three studies reported a statistically significant interaction between manganese exposure and sex $(p<0.05)$, without a clear pattern [41, 46, 48]. Rink et al. (2014) found that in children aged 14-45 months, $\mathrm{H}-\mathrm{Mn}$ was negatively associated with the cognitive, receptive language and expressive language scores for girls only in the unadjusted model [41]. While negative association between $\mathrm{H}-\mathrm{Mn}$ and the free recall after interference score was observed, especially in boys [46]. Chiu et al. (2017) found that higher prenatal Mn was associated with better body stability in boys, with opposite associations in girls. For tremor, on the other hand, higher early postnatal Mn was associated with increased right-hand center frequency in girls, but increased Mn concentration at the 
Table 4 Characteristics of the 18 studies that conducted sex-stratified analyses

\begin{tabular}{|c|c|c|c|c|c|c|}
\hline $\begin{array}{l}\text { Author, } \\
\text { Year }\end{array}$ & $\begin{array}{l}\text { Age } \\
\text { (Years) }\end{array}$ & Neurodevelopment & $\begin{array}{l}\text { Effect on } \\
\text { Boys }\end{array}$ & $\begin{array}{l}\text { Effect on } \\
\text { Girls }\end{array}$ & $\begin{array}{l}p \text {-value of } \\
\text { Interaction }\end{array}$ & Manganese Concentrations \\
\hline \multicolumn{7}{|c|}{ Neurodevelopmental outcomes from cohort studies } \\
\hline $\begin{array}{l}\text { Claus Henn } \\
2018[37]\end{array}$ & $6-16$ & Cognition & $\downarrow$ & NS & NA & Teeth: $12^{\mathrm{ab}}(n=138)$ \\
\hline $\begin{array}{l}\text { Dion } 2018 \\
{[20]}\end{array}$ & $10.5-18$ & Cognition & $\uparrow(\mathrm{W}-\mathrm{Mn})$ & $\downarrow(W-M n)$ & $<0.01(\mathrm{~W}-\mathrm{Mn})$ & $\begin{array}{l}\text { Drinking water: } 14.5 \mu \mathrm{g} / \mathrm{L}^{\mathrm{C}}(n=287) \text {, hair: } 1.4 \mu \mathrm{g} / \\
\mathrm{g}^{\mathrm{C}}(n=274)\end{array}$ \\
\hline $\begin{array}{l}\text { Gunier } 2015 \\
{[31]}\end{array}$ & $0.5,1,2$ & $\begin{array}{l}\text { Cognition and } \\
\text { motor }\end{array}$ & NS & $\downarrow$ & $\begin{array}{l}0.02 \text { (Cognition), } \\
0.03 \text { (Motor) }\end{array}$ & $\begin{array}{l}\text { Teeth: prenatal: } 0.51 \pm 0.19^{\text {bd }}(n=197), \text { postnatal: } \\
0.20 \pm 0.23^{\text {bd }}(n=193)\end{array}$ \\
\hline $\begin{array}{l}\text { Mora } 2015 \\
{[38]}\end{array}$ & $7,9,10.5$ & $\begin{array}{l}\text { Cognition and } \\
\text { motor }\end{array}$ & $\uparrow$ & NS & $<0.1$ & $\begin{array}{l}\text { Teeth: prenatal: } 0.50 \pm 0.18^{\text {bd }}(n=248) \text {, postnatal: } \\
0.19 \pm 0.21^{\text {bd }}(n=244)\end{array}$ \\
\hline $\begin{array}{l}\text { Mora } 2018 \\
{[33]}\end{array}$ & 1 & Cognition & NS & $\downarrow$ & 0.01 & $\begin{array}{l}\text { Maternal hair: } 3.7 \pm 5.4 \mu \mathrm{gg} / \mathrm{g}^{\mathrm{d}}(n=661) \text {, maternal } \\
\text { blood: } 24.4 \pm 6.2 \mu \mathrm{g} / \mathrm{L}^{\mathrm{d}}(n=571)\end{array}$ \\
\hline $\begin{array}{l}\text { Rahman } \\
2017 \text { [69] }\end{array}$ & 10 & Cognition & NS & $\uparrow$ & $<0.081$ & Drinking water: $339 \mu \mathrm{g} / \mathrm{L}^{\mathrm{a}}(n=1265)$ \\
\hline $\begin{array}{l}\text { Takser } 2003 \\
{[34]}\end{array}$ & Newborns & Motor & $\downarrow$ & NS & 0.03 & $\begin{array}{l}\text { Cord blood: } 38.5 \mu \mathrm{g} / \mathrm{L}^{\mathrm{c}}(n=222), \text { maternal blood: } \\
20.4 \mu \mathrm{g} / \mathrm{L}^{\mathrm{C}}(n=222), \text { maternal hair: } 0.36 \mu \mathrm{g} / \mathrm{g}^{\mathrm{c}} \\
(n=173), \text { newborns hair: } 0.75 \mu \mathrm{g} / \mathrm{g}^{\mathrm{c}}(n=173), \\
\text { placenta: } 0.1 \mu \mathrm{g} / \mathrm{g}^{\mathrm{c}}(n=200)\end{array}$ \\
\hline $\begin{array}{l}\text { Zhou } 2019 \\
\text { [39] }\end{array}$ & $6-8$ & Cognition & $\uparrow$ & NS & NA & $\begin{array}{l}\text { Cord blood: } 29.29 \pm 1.48 \mu \mathrm{g} / \mathrm{L}(n=296), \text { urine: } \\
0.66 \pm 3.81 \mu \mathrm{g} / \mathrm{L}(n=207)\end{array}$ \\
\hline \multicolumn{7}{|c|}{ Neurodevelopmental outcomes from cross-sectional studies } \\
\hline $\begin{array}{l}\text { Bauer } 2017 \\
{[42]}\end{array}$ & $10-14$ & Cognition & NS & $\downarrow$ & 0.05 & $\begin{array}{l}\text { Teeth: prenatal: } 0.42^{\mathrm{cb}}(n=142), \text { postnatal: } 0.12^{\mathrm{cb}} \\
(n=142)\end{array}$ \\
\hline $\begin{array}{l}\text { Bouchard } \\
2011[16]\end{array}$ & $6-13$ & Cognition & NS & $\downarrow$ & $\begin{array}{l}0.55(\mathrm{H}-\mathrm{Mn}), 0.14 \\
\text { (W-Mn) }\end{array}$ & $\begin{array}{l}\text { Hair: } 0.7 \mu \mathrm{g} / \mathrm{g}^{\mathrm{a}}(n=302) \text {, drinking water: } 0.8 \mu \mathrm{g} / \mathrm{L}^{\mathrm{a}} \\
(n=362)\end{array}$ \\
\hline $\begin{array}{l}\text { Bouchard } \\
2018[8]\end{array}$ & $6-14$ & Cognition & $\begin{array}{l}\text { NS (Toe nail), } \\
\uparrow(\mathrm{W}-\mathrm{Mn})\end{array}$ & $\begin{array}{l}\downarrow \text { (Toe nail), } \\
\text { NS (W-Mn) }\end{array}$ & $\begin{array}{l}0.028 \text { (Toe nail), } \\
0.015 \text { (W-Mn) }\end{array}$ & $\begin{array}{l}\text { Toe nail: } 2.0 \mu \mathrm{g} / \mathrm{g}^{c}(n=258) \text {, hair: } 0.3 \mu \mathrm{g} / \mathrm{g}^{c}(n= \\
\text { 258), saliva: } 1.1 \mu \mathrm{g} / \mathrm{L}^{\mathrm{c}}(n=226) \text {, drinking water: } \\
\left.\text { 5.2/7.3 } \mathrm{\mu g} / \mathrm{L}^{\mathrm{c}} \text { (boy: } 127, \text { girl: } 132\right)\end{array}$ \\
\hline $\begin{array}{l}\text { Carvalho } \\
2018[46]\end{array}$ & $7-12$ & Cognition & $\downarrow$ & NS & 0.047 & Hair: $11.5 \mu \mathrm{g} / \mathrm{g}^{\mathrm{a}}(n=70)$ \\
\hline $\begin{array}{l}\text { Chiu } 2017 \\
{[48]}\end{array}$ & $11-14$ & Motor & $\begin{array}{l}\text { NS (Prenatal } \\
\text { and } \\
\text { postnatal), } \downarrow \\
\text { (Childhood) }\end{array}$ & $\begin{array}{l}\downarrow \text { (Prenatal } \\
\text { and } \\
\text { postnatal), NS } \\
\text { (Childhood) }\end{array}$ & $\begin{array}{l}<0.05 \text { (Prenatal), } \\
<0.01 \\
\text { (Postnatal), } 0.01 \\
\text { (Childhood) }\end{array}$ & $\begin{array}{l}\text { Teeth: prenatal: } 0.43^{\mathrm{ab}}(n=189), \text { postnatal: } 0.13^{\mathrm{ab}} \\
(n=185)\end{array}$ \\
\hline $\begin{array}{l}\text { Hernandez- } \\
\text { Bonilla } 2016 \\
{[54]}\end{array}$ & $7-11$ & Cognition & NS & $\downarrow$ & $<0.15$ & $\begin{array}{l}\text { Hair: control: } 0.55 \mu \mathrm{g} / \mathrm{g}^{\mathrm{c}}(n=119) \text {, exposed: } \\
5.25 \mu \mathrm{g} / \mathrm{g}^{\mathrm{c}}(n=148)\end{array}$ \\
\hline $\begin{array}{l}\text { Menezes- } \\
\text { Filhoet } 2014 \\
{[62]}\end{array}$ & $7-12$ & Behavior & NS & $\downarrow$ & NA & $\begin{array}{l}\text { Hair: boys: } 12.1 \mu \mathrm{g} / \mathrm{g}^{\mathrm{a}}(n=34) \text {, girls: } 12.4 \mu \mathrm{g} / \mathrm{g}^{\mathrm{a}} \\
(n=36)\end{array}$ \\
\hline $\begin{array}{l}\text { Rink } 2014 \\
{[41]}\end{array}$ & $1.1-3.7$ & Cognition & $\uparrow$ & NS & $<0.05$ & Hair: $0.98 \pm 0.74 \mu \mathrm{g} / \mathrm{g}^{\mathrm{d}}(n=60)$ \\
\hline $\begin{array}{l}\text { Riojas- } \\
\text { Rodríguez } \\
2010[6]\end{array}$ & $7-11$ & Cognition & NS & $\downarrow$ & NA & $\begin{array}{l}\text { Control: hair: } 0.57 \mu \mathrm{g} / \mathrm{g} \text {, blood: } 8.22 \mu \mathrm{g} / \mathrm{L}^{\mathrm{c}}(n=93) \text {, } \\
\text { exposed: hair: } 12.13 \mu \mathrm{g} / \mathrm{g} \text {, blood: } 9.71 \mu \mathrm{g} / \mathrm{L}^{\mathrm{c}}(n= \\
\text { 79) }\end{array}$ \\
\hline $\begin{array}{l}\text { Torres- } \\
\text { Agustinet } \\
2013[67]\end{array}$ & $7-11$ & Cognition & NS & $\downarrow$ & NA & $\begin{array}{l}\text { Control: hair: } 0.6 \mu \mathrm{g} / \mathrm{g} \text {, blood: } 8.0 \mu \mathrm{g} / \mathrm{L}^{\mathrm{a}}(n=95) \\
\text { exposed: hair: } 12.6 \mu \mathrm{g} / \mathrm{g} \text {, blood:9.5 } \mathrm{\mu g} / \mathrm{L}^{\mathrm{a}}(n=79)\end{array}$ \\
\hline
\end{tabular}

$\downarrow$ : Negative association; $\uparrow$ : Positive association; NA Not available; NS No significant association. a: Median; b: ${ }^{55} \mathrm{Mn}:{ }^{43} \mathrm{Ca}$ the area under the curve (AUC) $\times 10^{4} ; \mathrm{c}$ : Geometric mean; d: Mean \pm standard deviation. H-Mn Manganese in hair; W-Mn Manganese in drinking water

later postnatal period was associated with increased center frequency in boys [48].

Three studies met the criteria for meta-analysis since they had a similar number of participants, and all adjusted for potential confounders such as maternal nonverbal intelligence, maternal education and family income $[8,16,20]$. Bouchard et al. (2011) only provided the detail of sex-stratified analysis for Full Scale IQ [16], therefore, there were two studies in the meta-analysis for Performance IQ and Verbal IQ. Figure 3 presents 


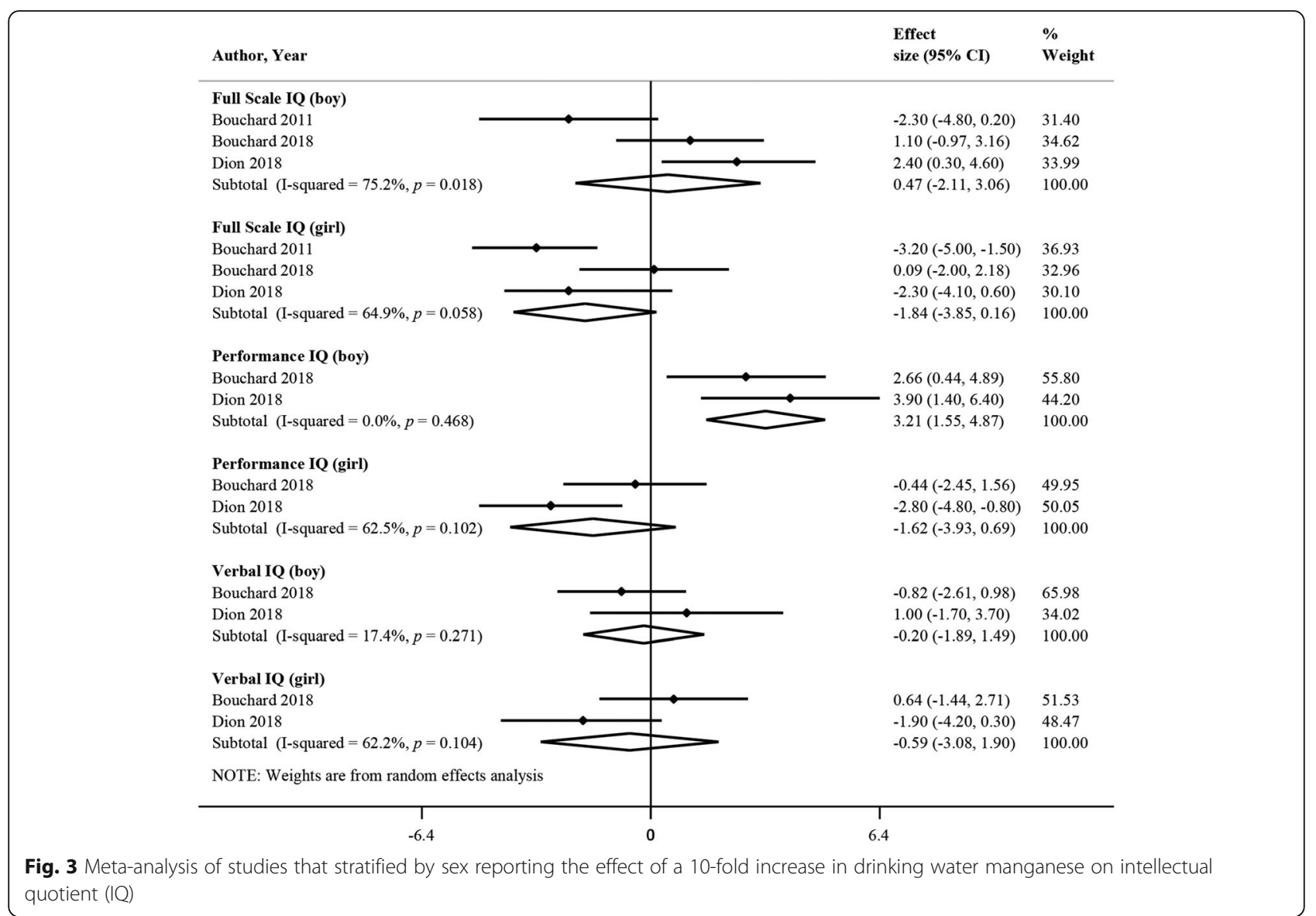

that higher $\mathrm{W}-\mathrm{Mn}$ is associated with better Performance IQ among boys only (change in scores for a 10-fold increase in concentration, $\beta=3.21 ; 95 \% \mathrm{CI}, 1.55,4.87$ ), in these two studies, a large percentage of children were exposed to drinking water manganese under $50 \mu \mathrm{g} / \mathrm{L}$ (the esthetic Canadian guideline concentration for W$\mathrm{Mn})[8,20]$. The meta-analysis on 3 studies concerning childhood IQ and $\mathrm{H}-\mathrm{Mn}$, found no significant difference between boys and girls $[8,16,20]$ (Additional file 8).

\section{Discussion}

This systemic review and meta-analysis was based on 55 studies, including 17 cohort studies and 38 crosssectional studies, with 13,388 participants. Evidence from cohort studies found that higher manganese exposure had a negative effect on neurodevelopment, mainly cognitive and motor skills in children under 6 years of age. In children aged 6-18 years old, results from crosssectional studies revealed that higher $\mathrm{H}-\mathrm{Mn}$ and $\mathrm{W}-\mathrm{Mn}$, but not B-Mn or T-Mn, were negatively associated with cognitive and behavioral performance. Of these crosssectional studies, most studies reported that the mean of manganese in hair was more than $0.55 \mu \mathrm{g} / \mathrm{g}$. The pooled results in $\mathrm{H}-\mathrm{Mn}$ revealed that a 10 -fold increase in $\mathrm{H}$ -
Mn was associated with a decrease of 2.51 points $(95 \%$ CI, - 4.58, - 0.45) in Full Scale IQ in children aged 618 years old. In the elder group, hair was the most consistent and reliable indicator of manganese exposure. These published data did demonstrate sex differences upon manganese exposure, without a clear pattern, possibly girls were more susceptible to manganese exposure than boys.

It is worth noting that the association between manganese exposure and motor performance was inconsistent in hair, blood and teeth. Only the results from infants $[27,34,36,70]$ and one study from the elder group that measured manganese in drinking water [15] supported the conclusion that higher manganese exposure had a negative effect on motor skills. It is a fact that occupational manganese exposure in adults can cause parkinsonian-like movement disorders [12]. Of note, animal studies reported that the increased brain manganese concentrations, either by $\mathrm{Mn}$ exposure or genetic strategies can cause severe motor deficits [73-75]. This consequence of excess Mn appears to be partly due to its interaction with other metals, like iron, operating through the post-transcriptional iron-responsive element driven regulatory mechanisms [76, 77]. Future studies 
are needed to evaluate the association between manganese exposure and motor performance in children.

Given the emerging evidence associating elevated $\mathrm{Mn}$ exposure with neurological impairments in children, it is critical to explore children's exposure to Mn from the different sources. Evidence from cross-sectional studies indicated that groundwater and industrial emissions from ferromanganese alloy plants and mining were the main sources of environmental manganese exposure. Therefore, children were exposed to manganese mainly by inhaling pollutants from industrial emissions and by drinking water. Compared to cross-sectional studies (Table 2), most birth cohort studies enrolled motherinfant pairs in hospitals or clinics without specific source of manganese exposure. Among these studies, they all measured manganese in biomarkers, which can reflect all sources (i.e. diet, air and water) and routes of exposures [78], although Mn homeostasis differs markedly for dietary uptake and inhalation. Additionally, almost all these studies carried out the analysis based on percentile grouping, with some studies yielding additional indication of a dose-response relation of any shape (i.e. linear or an inverted $U$ ). Nevertheless, further research is needed to explore the mechanism with respect to the absorption and distribution of different sources of manganese.

Additionally, there is a particular need for a consistent biomarker to accurately assess children's exposure to $\mathrm{Mn}$. The concentrations of manganese were frequently measured in hair and blood to reflect internal Mn dose in children aged 6-18 years. Results from water-borne and air-borne manganese exposure indicated that hair was more sensitive than blood to reflect the load of manganese in the body. What is more, hair manganese from airborne manganese exposure was much higher than waterborne manganese exposure and was negatively associated with childhood IQ scores.

From these analyses, hair is the more promising measure of long term $\mathrm{Mn}$ exposures when compared to blood (with a half-life of 4 or 39 days due to the different elimination pathways [79]). Many metals are deposited in keratin, a component of hair, and the relatively slow growth rate of hair means that hair represents integrated exposures [80]. The $2 \mathrm{~cm}$ of newly grown hair was used for measuring the concentration of manganese in most publications, which reflects the exposure during the 2-4 months before sampling [81]. In addition, teeth also reflects long-term exposures as a slow metabolism and accumulation of Mn occurs in teeth [82]. Among eight studies that used teeth as a biomarker, all of these studies measured manganese in naturally shed deciduous milk teeth, while the teeth type varied among these studies, such as for incisors, canines and molars. In most studies, cumulative $\mathrm{Mn}$ exposures were estimated in incisors that were free of obvious defects such as caries and extensive tooth wear, which reflect manganese exposure from 13 to 16 weeks after gestation to approximately 1 year of age [83]. In fact, animal studies showed that H-Mn was significantly correlated with T-Mn. Furthermore, correlation coefficients clearly supported links between $\mathrm{H}-\mathrm{Mn}$ and cognitive functions, reflected by escape latencies and number of platform crossings, and the correlations were better than those in teeth [84]. Additionally, hair is easier to obtain than teeth. Toe-nail can also be employed as a tissue source to measure of chronic exposure to this metal [8], but this technique has rarely been used when establishing environmental manganese exposures in children. The characteristics of relevant biomarkers are summarized in Table $5[79,81-$ 83, 85-99].

Overall, this review suggests that hair is the most reliable indicator of environmental manganese exposure in children aged 6-18 years old. Traditionally, the main problem of using hair as a biomarker is the potential for external contamination. In response to this, except for a study published in 2007 [45], all other studies that measured manganese in hair had used defined cleaning methodologies to eliminate external contamination. Eastman et al. (2013), for example, developed a hair cleaning methodology to effectively eliminate exogenous metal contamination [86]. This method can substantiate the use of hair as a biomarker of environmental Mn exposure in children. It should be noted that hair dye or other topical treatment could influence the content of manganese in hair [100], although topical hair treatment is unfrequent in children. In spite of this, two studies, now included, also excluded children who reported using hair dye in the preceding 5 months $[15,16]$. Further work is needed to determine the utility of hair as a biomarker in preschoolers exposed to manganese. For infants, there appears to be insufficient hair to be analyzed. It is worth noting that teeth provides integrated measures of exposure over the prenatal and early childhood periods of their development, perhaps presenting as a promising biomarker of manganese exposure in infants.

It is always important to accurately determine the safe range of manganese exposure. For this reason, we extracted the reference range or cut-off point used in the reviewed articles (see Additional file 9), while limitations in our data precluded us from directly addressing some aspects of this important issue. In regard to $\mathrm{H}-\mathrm{Mn}$, we found that the cut-off point was much higher than the upper limit value of reference range. Accordingly, negative associations between $\mathrm{H}-\mathrm{Mn}$ and neurodevelopment were observed in two studies that used 2 or $3 \mu \mathrm{g} / \mathrm{g}$ as the cut-off point [43, 45]. And Haynes et al. (2015) found that compared with $0.21-0.75 \mu \mathrm{g} / \mathrm{g}$, both lower 
Table 5 Characteristics of relevant biomarkers used in children

\begin{tabular}{|c|c|c|c|}
\hline Biomarkers & Characteristics & Advantages & Limitations \\
\hline Hair & $\begin{array}{l}\text { Reflects the exposure during the } 2-4 \\
\text { months before sampling [81] }\end{array}$ & $\begin{array}{l}\text { Easy to collect, store and manipulate, non- } \\
\text { invasive, most consistent and valid biomarker in } \\
\text { pediatric epidemiology [85] }\end{array}$ & $\begin{array}{l}\text { Pigmentation and potential external } \\
\text { contamination [86] }\end{array}$ \\
\hline Blood & $\begin{array}{l}\text { With a half-life of } 4 \text { or } 39 \text { days due to } \\
\text { different elimination pathways [79] }\end{array}$ & $\begin{array}{l}\text { Obtained easily and less influence of external } \\
\text { contamination [87] }\end{array}$ & Correlated poorly with exposure [88] \\
\hline Teeth & $\begin{array}{l}\text { Reflects the exposure from } 13 \text { to } 16 \\
\text { weeks after gestation to approximately } \\
\text { one year of age [83] }\end{array}$ & $\begin{array}{l}\text { Non-invasive, provides precise exposure } \\
\text { information, distinguishes the prenatal and } \\
\text { postnatal exposure [82] }\end{array}$ & $\begin{array}{l}\text { Caries and teeth with attrition contained } \\
\text { less metal [89], relatively difficult to } \\
\text { obtain and measure }\end{array}$ \\
\hline $\begin{array}{l}\text { Saliva and } \\
\text { urine }\end{array}$ & $\begin{array}{l}\text { secretes } 0.8 \text { to } 1.5 \mathrm{~L} \text { of saliva each day, } \\
\text { only a small fraction of Mn eliminates in } \\
\text { urine [90] }\end{array}$ & Non-invasive and easy to collect [90] & $\begin{array}{l}\text { Correlated poorly with exposure }[88,91 \text {, } \\
\text { 92], fairly large variation [93] }\end{array}$ \\
\hline Toe nail & $\begin{array}{l}\text { Reflects an exposure of } 7-12 \text { months } \\
\text { before sampling }[94,95]\end{array}$ & $\begin{array}{l}\text { Easy collection, storage and transport [96], } \\
\text { correlated with exposure [91] }\end{array}$ & $\begin{array}{l}\text { Difficult to collect sufficient toenail from } \\
\text { infants and potential external } \\
\text { contamination [97] }\end{array}$ \\
\hline Cord blood & $\begin{array}{l}\text { Reflects an exposure of the last trimester } \\
\text { [97] }\end{array}$ & Correlated with manganese in dentin [98] & $\begin{array}{l}\text { Not feasible to obtain at different stages } \\
\text { of pregnancy [98] }\end{array}$ \\
\hline $\begin{array}{l}\text { Maternal } \\
\text { blood }\end{array}$ & $\begin{array}{l}\text { Mn enters the fetus via an active } \\
\text { transport mechanism [99] }\end{array}$ & Readily sampled [98] & $\begin{array}{l}\text { Maternal Mn biomarkers may not } \\
\text { accurately reflect Mn levels in fetal } \\
\text { tissues [98] }\end{array}$ \\
\hline
\end{tabular}

Mn Manganese

and higher $\mathrm{H}-\mathrm{Mn}$ were associated with lower IQ scores [52], this may be closer to the possible reference range in children.

It will be critical to consider the timing of Mn exposures, because there may be certain sensitive periods to the effects of environmental manganese exposures in the developing brain. Takser et al. (2003) found that there were negative relationships between cord blood Mn concentrations and several psychomotor sub-scales at age of 3 years, but not at 9 months or 6 years, after adjustment for potential confounders [34].

Some included studies measured prenatal exposure, as indicated by manganese in maternal and cord blood, maternal and infant hair and placenta. Other studies measured manganese in teeth, which reflects prenatal and postnatal exposure (from 13 to 16 weeks after gestation to 1 year of age). Most of the included studies measured postnatal manganese exposure with a cross-sectional design. Hair was the frequently-used biomarker, where that analyzed $2 \mathrm{~cm}$ closest to the scalp reflects the exposure during the 2-4 months before sampling [81]. Among these studies, we tend to believe that manganese exposure is continuous, as some cross-sectional studies recruited children who had lived in the same community for a minimum of 3 months or 5 years, to ensure continuous exposure to the same source for this period of time. The follow-up study is warranted to explore the periods of critical vulnerability of environmental manganese exposures.

In this review, information regarding sex differences of manganese exposure from both cohort studies and cross-sectional studies were inconsistent. Perhaps there was a trend showing that girls were more susceptible to manganese exposure than boys. While almost all studies found no significant sex differences for $\mathrm{Mn}$ in biomarkers and drinking water, except for four studies without the relevant details available [34, 39, 54, 67]. Given that most studies were not specifically designed to evaluate sex-interactions, therefore, low statistical power may in part explain some of the inconsistency between studies.

Recently a study of single nucleotide polymorphisms in $\mathrm{Mn}$ transporter genes SLC30A10 and SLC39A8 also found a sex difference between $\mathrm{Mn}$ concentrations and genotypes [101]. The mechanisms behind potential sex differences in Mn toxicity are complicated, possibly due to sex difference in the developing brain [102], possibly related to biological differences in neurochemistry and hormone activity [103]. In addition, data from animal studies had shown that $\mathrm{Mn}$ exposure caused sexdependent neuronal morphological change, and this change was not due to differential Mn accumulation between sexes but due to differences in sensitivity to $\mathrm{Mn}$ exposure [104]. All these differences may contribute to sex dimorphism in the associations between Mn exposure and neurodevelopment.

Our study incorporated the following limitations that warrant discussion. Firstly, most studies in this review are cross-sectional studies, so that no causal relationship can be inferred. In addition, a consistent biomarker for infants was not identified, perhaps teeth is the most promising biosample in this case, while being less easy to obtain than hair. Limitations in our data precluded us from identifying the safe range of manganese exposure 
and the periods of critical vulnerability of environmental manganese exposures. Finally, limited number of studies could be analyzed due to the relative homogeneity, however, we do not believe that this affected our analysis, given the stability of our sensitivity analysis.

Overall, to the best of our knowledge, this is the only comprehensive systemic review and meta-analysis regarding the biomarkers and sources of manganese exposure and cognitive, behavioral and motor functions in children. Outcomes from cohort studies and crosssectional studies indicated that higher manganese exposures were negatively associated with neurodevelopment in children. In addition, this is the first meta-analysis for correlation between different manganese indicators where our results indicated that $\mathrm{H}-\mathrm{Mn}$ was more significantly correlated with W-Mn than B-Mn. Therefore, we propose that hair is the most suitable biomarker in future studies.

\section{Conclusions}

Higher manganese exposure is negatively associated with childhood neurodevelopment, especially cognitive and motor skills for children under 6 years old and cognitive and behavioral performance for children aged 6-18 years old. In the older group (6-18 years old), hair is the most reliable indicator of manganese exposure. However, evidence demonstrated sex difference upon manganese exposure while a clear pattern is not elucidated. Population based biomonitoring studies with standard cleaning methodologies of hair are warranted in order to set reference ranges of manganese in hair at different ages. Large prospective cohort studies are certainly warranted in order to support these results and identify the underlying biological mechanisms.

\section{Supplementary information}

Supplementary information accompanies this paper at https://doi.org/10. 1186/s12940-020-00659-X.

\section{Additional file 1. PRISMA 2009 Checklist}

Additional file 2. Evaluation of methodological quality of articles by using checklist in the Strengthening the Reporting of Observational Studies in Epidemiology Statement

Additional file 3. Characteristics of the articles included in the metaanalysis

Additional file 4. Sensitivity analysis was performed to evaluate the stability of the result

Additional file 5. Meta-analysis of studies reporting the effect of a 10fold increase in drinking water manganese on intellectual quotient (IQ)

Additional file 6. Meta-analysis of studies reporting the effect of a efold increase in blood manganese on intellectual quotient (IQ)

Additional file 7. Correlations between manganese in biomarkers and environmental sample

Additional file 8. Meta-analysis of studies that stratified by sex reporting the effect of a 10-fold increase in hair manganese on intellectual quotient (IQ)
Additional file 9. The reference range or cut-off point used in the reviewed articles

\section{Abbreviations}

AARES: The academic achievement records of the elementary schools; ADS: The attention-deficit/hyperactivity disorder (ADHD) diagnostic system; AMP: Aptitudes mentales primarias; APS: Accusway plus system; BASC2: Behavior assessment system for children, 2nd edition; BOT-2: The bruininks-oseretsky test, 2nd edition; BSID: Bayley scales of infant and toddler development; CANTAB: Cambridge neuropsychological test automated battery; CAVLT: The children's auditory verbal learning test; CBCL: The standardized child behavior checklist; CDIIT: The comprehensive developmental inventory for infants and toddlers; CPRS-R: The revised conners' rating scale for parents; CPT-II: Conners' continuous performance test ii version; CTRS-R: The revised conners' rating scale for teachers; DBD: The disruptive behavior disorders; DDST-II: Denver developmental screening test II; DPD: Danish products developments; DS: Digit span; FT: Finger tapping; FTT: The forbidden toy task; GDI: Gesell developmental inventory; GP: Grooved pegboard; LNMB: Luria nebraska motor battery; MSCA: The McCarthy scales of children's abilities; NBNA: Neonatal behavioral neurological assessments; NEPSY-II: Developmental neuropsychological assessment, second edition; NEUPSILIN-Inf: The Brazilian child brief neuropsychological assessment battery; PA: Pursuit aiming; PCM: The Raven's progressive color matrices scale; PEDS: Parents' evaluation of developmental status; RCPM: The Raven's Colored progressive matrices; ROCF: The reyosterrieth complex fig.; SA: Santa ana test; SDQ: The strengths and difficulties questionnaire; VRAM: The virtual radial arm maze; WASI: Wechsler abbreviated scale of intelligence; WISC: The wechsler intelligence scale for children; W-M: Woodcock-Muñoz tests of cognitive abilities; WRAVMA: The wide range assessment of visual motor abilities

\section{Acknowledgments}

We thank the members of the Wang and Min Laboratories for helpful discussions, especially Junhao Wang, Hao Wang, Xuexian Fang and Peng An.

\section{Authors' contributions}

W.L., Y.X., F.W. and J.T.R. designed the study; W.L., Y.X.and Q. L identified the studies for inclusion, extracted the data and assessed the quality of the included studies; W.L. and Q.L. conducted the meta-analysis; W.L. and Y.X. wrote the first draft of the manuscript; Y.S. and Z.P. provided critical input for the manuscript; F.W., J.T.R., J.M. and C.M.C. did critical revision of the manuscript to improve and optimally present the key intellectual content and they also supervised this study. All authors have contributed significantly, and all authors are in agreement with respect to the content of the manuscript. The author (s) read and approved the final manuscript.

\section{Funding}

This research was funded by the National Natural Science Foundation of China (31530034 and 31930057 to F.W.; 31570791 to J.M.) and the National Key Research and Development Program of China (2018YFA0507802 to F.W.; 2018YFA0507801 to J.M.), and Michael J. FOX Foundation (J.T.R.).

Ethics approval and consent to participate

No human subjects, human material, or human data were involved in this research, which is based on literature review.

Consent for publication

Not applicable.

Competing interests

The authors declare that they have no competing interests.

\section{Author details}

${ }^{1}$ Department of Nutrition, Precision Nutrition Innovation Center, School of Public Health, Zhengzhou University, Zhengzhou, China. ${ }^{2}$ The First Affiliated Hospital, School of Public Health, Institute of Translational Medicine, Zhejiang University School of Medicine, Hangzhou, China. ${ }^{3}$ Neurochemistry Laboratory, Department of Psychiatry-Neuroscience, Massachusetts General Hospital and Harvard Medical School, Charlestown, MA, USA. 
Received: 1 July 2020 Accepted: 22 September 2020

Published online: 02 October 2020

\section{References}

1. Aschner M, Erikson K. Manganese. Adv Nutr. 2017;8(3):520-1.

2. de Water E, Proal E, Wang V, Medina SM, Schnaas L, Tellez-Rojo MM, et al. Prenatal manganese exposure and intrinsic functional connectivity of emotional brain areas in children. Neurotoxicology. 2018;64:85-93.

3. Leonhard MJ, Chang ET, Loccisano AE, Garry MR. A systematic literature review of epidemiologic studies of developmental manganese exposure and neurodevelopmental outcomes. Toxicology. 2019:420:46-65.

4. lyare PU. The effects of manganese exposure from drinking water on school-age children: a systematic review. Neurotoxicology. 2019:73:1-7.

5. Saghazadeh A, Rezaei N. Systematic review and meta-analysis links autism and toxic metals and highlights the impact of country development status: higher blood and erythrocyte levels for mercury and lead, and higher hair antimony, cadmium, lead, and mercury. Prog Neuropsychopharmacol Biol Psychiatry. 2017;79(Pt B):340-68

6. Riojas-Rodríguez H, Solís-Vivanco R, Schilmann A, Montes S, Rodríguez S, Ríos $C$, et al. Intellectual function in Mexican children living in a mining area and environmentally exposed to manganese. Environ Health Perspect. 2010; 118(10):1465-70.

7. Haynes EN, Sucharew H, Hilbert TJ, Kuhnell P, Spencer A, Newman NC, et al. Impact of air manganese on child neurodevelopment in East Liverpool, Ohio. Neurotoxicology. 2018;64:94-102.

8. Bouchard MF, Surette C, Cormier P, Foucher D. Low level exposure to manganese from drinking water and cognition in school-age children. Neurotoxicology. 2018;64:110-7.

9. Wasserman GA, Liu X, Parvez F, Ahsan H, Levy D, Factor-Litvak P, et al. Water manganese exposure and children's intellectual function in Araihazar, Bangladesh. Environ Health Perspect. 2006;114(1):124-9.

10. Rodriguez-Barranco M, Lacasana M, Aguilar-Garduno C, Alguacil J, Gil F, Gonzalez-Alzaga B, et al. Association of arsenic, cadmium and manganese exposure with neurodevelopment and behavioural disorders in children: a systematic review and meta-analysis. Sci Total Environ. 2013:454-455:562-77.

11. Meyer-Baron M, Knapp G, Schäper M, van Thriel C. Performance alterations associated with occupational exposure to manganese--a meta-analysis. Neurotoxicology. 2009;30(4):487-96.

12. Kwakye GF, Paoliello MM, Mukhopadhyay S, Bowman AB, Aschner M. Manganese-induced parkinsonism and Parkinson's disease: shared and distinguishable features. Int J Env Res Pub He. 2015;12(7):7519-40.

13. Llop S, Lopez-Espinosa MJ, Rebagliato M, Ballester F. Gender differences in the neurotoxicity of metals in children. Toxicology. 2013;311(1-2):3-12.

14. Shih JH, Zeng BY, Lin PY, Chen TY, Chen YW, Wu CK, et al. Association between peripheral manganese levels and attention-deficit/hyperactivity disorder: a preliminary meta-analysis. Neuropsychiatr Dis Treat. 2018;14: 1831-42.

15. Oulhote $Y$, Mergler D, Barbeau B, Bellinger DC, Bouffard T, Brodeur ME, et al Neurobehavioral function in school-age children exposed to manganese in drinking water. Environ Health Perspect. 2014;122(12):1343-50.

16. Bouchard MF, Sauve S, Barbeau B, Legrand M, Brodeur ME, Bouffard T, et al. Intellectual impairment in school-age children exposed to manganese from drinking water. Environ Health Perspect. 2011;119(1):138-43.

17. von Elm E, Altman DG, Egger M, Pocock SJ, Gøtzsche PC, Vandenbroucke JP. The strengthening the reporting of observational studies in epidemiology (STROBE) statement: guidelines for reporting observational studies. Int J Surg. 2014;12(12):1495-9.

18. Mikó A, Pótó L, Mátrai P, Hegyi P, Füredi N, Garami A, et al. Gender difference in the effects of interleukin-6 on grip strength - a systematic review and meta-analysis. BMC Geriatr. 2018;18(1):107.

19. Wasserman GA, Liu X, Parvez F, Factor-Litvak P, Kline J, Siddique AB, et al. Child intelligence and reductions in water arsenic and manganese: a twoyear follow-up study in Bangladesh. Environ Health Perspect. 2016;124(7): 1114-20.

20. Dion LA, Saint-Amour D, Sauve S, Barbeau B, Mergler D, Bouchard MF. Changes in water manganese levels and longitudinal assessment of intellectual function in children exposed through drinking water. Neurotoxicology. 2018;64:118-25.

21. Menezes-Filho JA, Novaes Cde O, Moreira JC, Sarcinelli PN, Mergler D. Elevated manganese and cognitive performance in school-aged children and their mothers. Environ Res. 2011;111(1):156-63.
22. Carvalho CF, Menezes-Filho JA, de Matos VP, Bessa JR, Coelho-Santos J, Viana GF, et al. Elevated airborne manganese and low executive function in school-aged children in Brazil. Neurotoxicology. 2014;45:301-8.

23. Wechsler D. Wechsler intelligence scale for children. San Antonio, TX: Psychological Corporation; 1991.

24. Shadish WR, Haddock CK. Combining estimates of effect size. In: Cooper H, Hedges LV, editors. The Handbook of Research Synthesis. New York: Russell Sage Foundation; 1994. p. 265-6.

25. Higgins JP, Thompson SG, Deeks JJ, Altman DG. Measuring inconsistency in meta-analyses. BMJ. 2003;327(7414):557-60.

26. Egger M, Davey Smith G, Schneider M, Minder C. Bias in meta-analysis detected by a simple, graphical test. BMJ. 1997;315(7109):629-34.

27. Chung SE, Cheong HK, Ha EH, Kim BN, Ha M, Kim Y, et al. Maternal blood manganese and early neurodevelopment: the mothers and children's environmental health (MOCEH) study. Environ Health Perspect. 2015;123(7): 717-22.

28. Claus Henn B, Ettinger AS, Schwartz J, Tellez-Rojo MM, Lamadrid-Figueroa H, Hernandez-Avila M, et al. Early postnatal blood manganese levels and children's neurodevelopment. Epidemiology. 2010;21(4):433-9.

29. Claus Henn B, Bellinger DC, Hopkins MR, Coull BA, Ettinger AS, Jim R, et al Maternal and cord blood manganese concentrations and early childhood neurodevelopment among residents near a mining-impacted superfund site. Environ Health Perspect. 2017;125(6):067020.

30. Freire C, Amaya E, Gil F, Fernandez MF, Murcia M, Llop S, et al. Prenatal coexposure to neurotoxic metals and neurodevelopment in preschool children: the environment and childhood (INMA) project. Sci Total Environ. 2018;621:340-51.

31. Gunier RB, Arora M, Jerrett M, Bradman A, Harley KG, Mora AM, et al. Manganese in teeth and neurodevelopment in young Mexican-American children. Environ Res. 2015:142:688-95.

32. Lin CC, Chen YC, Su FC, Lin CM, Liao HF, Hwang YH, et al. In utero exposure to environmental lead and manganese and neurodevelopment at 2 years of age. Environ Res. 2013;123:52-7.

33. Mora AM, Cordoba L, Cano JC, Hernandez-Bonilla D, Pardo L, Schnaas L, et al. Prenatal mancozeb exposure, excess manganese, and neurodevelopment at 1 year of age in the infants' environmental health (ISA) study. Environ Health Perspect. 2018;126(5):057007.

34. Takser L, Mergler D, Hellier G, Sahuquillo J, Huel G. Manganese, monoamine metabolite levels at birth, and child psychomotor development. Neurotoxicology. 2003;24(4-5):667-74.

35. Yu XD, Zhang J, Yan $\mathrm{CH}$, Shen XM. Prenatal exposure to manganese at environment relevant level and neonatal neurobehavioral development. Environ Res. 2014:133.232-8.

36. Yu X, Chen L, Wang C, Yang X, Gao Y, Tian Y. The role of cord blood BDNF in infant cognitive impairment induced by low-level prenatal manganese exposure: LW birth cohort, China. Chemosphere. 2016;163:446-51.

37. Claus Henn B, Austin C, Coull BA, Schnaas L, Gennings C, Horton MK, et al, Uncovering neurodevelopmental windows of susceptibility to manganese exposure using dentine microspatial analyses. Environ Res. 2018:161:588-98.

38. Mora AM, Arora M, Harley KG, Kogut K, Parra K, Hernandez-Bonilla D, et al. Prenatal and postnatal manganese teeth levels and neurodevelopment at 7 , 9, and 10.5 years in the CHAMACOS cohort. Environ Int. 2015;84:39-54.

39. Zhou T, Guo J, Zhang J, Xiao H, Qi X, Wu C, et al. Sex-specific differences in cognitive abilities associated with childhood cadmium and manganese exposures in school-age children: a prospective cohort study. Biol Trace Elem Res. 2019;193(1):89-99.

40. Al-Saleh I, Al-Mohawes S, Al-Rouqi R, Elkhatib R. Selenium status in lactating mothers-infants and its potential protective role against the neurotoxicity of methylmercury, lead, manganese, and DDT. Environ Res. 2019;176:108562.

41. Rink SM, Ardoino G, Queirolo El, Cicariello D, Manay N, Kordas K. Associations between hair manganese levels and cognitive, language, and motor development in preschool children from Montevideo, Uruguay. Arch Environ Occup Health. 2014;69(1):46-54

42. Bauer JA, Claus Henn B, Austin C, Zoni S, Fedrighi C, Cagna G, et al. Manganese in teeth and neurobehavior: sex-specific windows of susceptibility. Environ Int. 2017;108:299-308.

43. Betancourt O, Tapia M, Mendez I. Decline of general intelligence in children exposed to manganese from mining contamination in puyango river basin, southern Ecuador. Ecohealth. 2015;12(3):453-60.

44. Bhang SY, Cho SC, Kim JW, Hong YC, Shin MS, Yoo HJ, et al. Relationship between blood manganese levels and children's attention, cognition, 
behavior, and academic performance--a nationwide cross-sectional study. Environ Res. 2013;126:9-16.

45. Bouchard M, Laforest F, Vandelac L, Bellinger D, Mergler D. Hair manganese and hyperactive behaviors: pilot study of school-age children exposed through tap water. Environ Health Perspect. 2007;115(1):122-7.

46. Carvalho CF, Oulhote Y, Martorelli M, Carvalho CO, Menezes-Filho JA, Argollo N, et al. Environmental manganese exposure and associations with memory, executive functions, and hyperactivity in Brazilian children. Neurotoxicology. 2018;69:253-9.

47. Chan TJ, Gutierrez C, Ogunseitan OA. Metallic burden of deciduous teeth and childhood behavioral deficits. Int J Environ Res Pub He 2015;12(6):6771-87.

48. Chiu YM, Claus Henn B, Hsu HL, Pendo MP, Coull BA, Austin C, et al. Sex differences in sensitivity to prenatal and early childhood manganese exposure on neuromotor function in adolescents. Environ Res. 2017;159: 458-65.

49. do Nascimento SN, Barth A, Goethel G, Baierle M, Charao MF, Brucker N, et al. Cognitive deficits and ALA-D-inhibition in children exposed to multiple metals. Environ Res. 2015;136:387-95.

50. Ericson JE, Crinella FM, Clarke-Stewart KA, Allhusen VD, Chan T, Robertson RT. Prenatal manganese levels linked to childhood behavioral disinhibition. Neurotoxicol Teratol. 2007;29(2):181-7.

51. Frndak S, Barg G, Canfield RL, Quierolo El, Manay N, Kordas K. Latent subgroups of cognitive performance in lead- and manganese-exposed Uruguayan children: examining behavioral signatures. Neurotoxicology. 2019:73:188-98

52. Haynes EN, Sucharew H, Kuhnell P, Alden J, Barnas M, Wright RO, et al. Manganese exposure and neurocognitive outcomes in rural school-age children: the communities actively researching exposure study (Ohio, USA) Environ Health Perspect. 2015;123(10):1066-71.

53. Hernandez-Bonilla D, Schilmann A, Montes S, Rodriguez-Agudelo $Y$, Rodriguez-Dozal S, Solis-Vivanco R, et al. Environmental exposure to manganese and motor function of children in Mexico. Neurotoxicology. 2011;32(5):615-21.

54. Hernandez-Bonilla D, Escamilla-Nunez C, Mergler D, Rodriguez-Dozal S, Cortez-Lugo M, Montes S, et al. Effects of manganese exposure on visuoperception and visual memory in schoolchildren. Neurotoxicology. 2016;57:230-40

55. Horton MK, Hsu L, Claus Henn B, Margolis A, Austin C, Svensson K, et al. Dentine biomarkers of prenatal and early childhood exposure to manganese, zinc and lead and childhood behavior. Environ Int. 2018;121(Pt 1):148-58.

56. Khan K, Factor-Litvak P, Wasserman GA, Liu X, Ahmed E, Parvez F, et al. Manganese exposure from drinking water and children's classroom behavior in Bangladesh. Environ Health Perspect. 2011;119(10):1501-6.

57. Kicinski $M$, Vrijens J, Vermier $G$, Hond ED, Schoeters G, Nelen V, et al. Neurobehavioral function and low-level metal exposure in adolescents. Int $J$ Hyg Environ Health. 2015;218(1):139-46.

58. Kim Y, Kim BN, Hong YC, Shin MS, Yoo HJ, Kim JW, et al. Co-exposure to environmental lead and manganese affects the intelligence of school-aged children. Neurotoxicology. 2009;30(4):564-71.

59. Lucchini RG, Zoni S, Guazzetti S, Bontempi E, Micheletti S, Broberg K, et al. Inverse association of intellectual function with very low blood lead but not with manganese exposure in Italian adolescents. Environ Res. 2012a;1 18:65-71.

60. Lucchini RG, Guazzetti S, Zoni S, Donna F, Peter S, Zacco A, et al. Tremor, olfactory and motor changes in Italian adolescents exposed to historical ferro-manganese emission. Neurotoxicology. 2012b;33(4):687-96.

61. Lucchini RG, Guazzetti S, Renzetti S. Neurocognitive impact of metal exposure and social stressors among schoolchildren in Taranto, Italy. Environ Health. 2019:18(1):67.

62. Menezes-Filho JA, de Carvalho-Vivas CF, Viana GF, Ferreira JR, Nunes LS, Mergler D, et al. Elevated manganese exposure and school-aged children's behavior: a gender-stratified analysis. Neurotoxicology. 2014;45:293-300.

63. Nascimento S, Baierle M, Goethel G, Barth A, Brucker N, Charao M, et al. Associations among environmental exposure to manganese, neuropsychological performance, oxidative damage and kidney biomarkers in children. Environ Res. 2016;147:32-43.

64. Parvez F, Wasserman GA, Factor-Litvak P, Liu X, Slavkovich V, Siddique AB, et al. Arsenic exposure and motor function among children in Bangladesh Environ Health Perspect. 2011;119(11):1665-70.

65. Rugless F, Bhattacharya A, Succop P, Dietrich KN, Cox C, Alden J, et al. Childhood exposure to manganese and postural instability in children living near a ferromanganese refinery in southeastern Ohio. Neurotoxicol Teratol. 2014;41:71-9.

66. Torrente M, Colomina MT, Domingo JL. Metal concentrations in hair and cognitive assessment in an adolescent population. Biol Trace Elem Res. 2005;104(3):215-21.

67. Torres-Agustin R, Rodriguez-Agudelo Y, Schilmann A, Solis-Vivanco R, Montes S, Riojas-Rodriguez H, et al. Effect of environmental manganese exposure on verbal learning and memory in Mexican children. Environ Res. 2013;121:39-44.

68. Wright RO, Amarasiriwardena C, Woolf AD, Jim R, Bellinger DC. Neuropsychological correlates of hair arsenic, manganese, and cadmium levels in school-age children residing near a hazardous waste site. Neurotoxicology. 2006;27(2):210-6.

69. Rahman SM, Kippler M, Tofail F, Bolte S, Hamadani JD, Vahter M. Manganese in drinking water and cognitive abilities and behavior at 10 years of age: a prospective cohort study. Environ Health Perspect. 2017;125(5):057003.

70. Rodrigues EG, Bellinger DC, Valeri L, Hasan MO, Quamruzzaman Q, Golam $\mathrm{M}$, et al. Neurodevelopmental outcomes among 2- to 3-year-old children in Bangladesh with elevated blood lead and exposure to arsenic and manganese in drinking water. Environ Health. 2016;15:44.

71. Khan K, Wasserman GA, Liu X, Ahmed E, Parvez F, Slavkovich V, et al. Manganese exposure from drinking water and children's academic achievement. Neurotoxicology. 2012;33(1):91-7.

72. Wasserman GA, Liu X, Parvez F, Factor-Litvak P, Ahsan H, Levy D, et al. Arsenic and manganese exposure and children's intellectual function. Neurotoxicology. 2011;32(4):450-7.

73. Tuschl K, Meyer E, Valdivia LE, Zhao N, Dadswell C, Abdul-Sada A, et al. Mutations in SLC39A14 disrupt manganese homeostasis and cause childhood-onset parkinsonism-dystonia. Nat Commun. 2016;7:11601.

74. Xin Y, Gao H, Wang J, Qiang Y, Imam MU, Li Y, et al. Manganese transporter slc39a14 deficiency revealed its key role in maintaining manganese homeostasis in mice. Cell Discov. 2017;3:17025.

75. Xia Z, Wei J, Li Y, Wang J, Li W, Wang K, et al. Zebrafish slc30a10 deficiency revealed a novel compensatory mechanism of Atp2c1 in maintaining manganese homeostasis. PLoS Genet. 2017;13(7):e1006892.

76. Rogers JT, Xia N, Wong A, Bakshi R, Cahill CM. Targeting the iron-response elements of the mRNAs for the Alzheimer's amyloid precursor protein and ferritin to treat acute lead and manganese neurotoxicity. Int J Mol Sci. 2019; 20(4):994.

77. Venkataramani V, Doeppner TR, Willkommen D, Cahill CM, Xin Y, Ye G, et al. Manganese causes neurotoxic iron accumulation via translational repression of amyloid precursor protein and H-ferritin. J Neurochemistry. 2018;147(6): $831-48$

78. Aprea MC. Environmental and biological monitoring in the estimation of absorbed doses of pesticides. Toxicol Lett. 2012;210(2):110-8.

79. Mahoney JP, Small WJ. Studies on manganese. 3. The biological half-life of radiomanganese in man and factors which affect this half-life. I Clin Invest. 1968:47(3):643-53

80. Kordas K, Queirolo El, Ettinger AS, Wright RO, Stoltzfus RJ. Prevalence and predictors of exposure to multiple metals in preschool children from Montevideo, Uruguay. Sci Total Environ. 2010;408(20):4488-94.

81. Robbins CR. Chemical and physical behavior of human hair. 4th ed. New York: Springer-Verlag; 2002.

82. Arora M, Hare D, Austin C, Smith DR, Doble P. Spatial distribution of manganese in enamel and coronal dentine of human primary teeth. $\mathrm{Sci}$ Total Environ. 2011;409(7):1315-9.

83. Hillson S. Dental anthropology. New York: Cambridge University Press; 1996.

84. Liang G, Zhang L, Ma S, Lv Y, Qin H, Huang $X$, et al. Manganese accumulation in hair and teeth as a biomarker of manganese exposure and neurotoxicity in rats. Environ Sci Pollut Res Int. 2016;23(12):1226571.

85. Coetzee DJ, McGovern PM, Rao R, Harnack LJ, Georgieff MK, Stepanov I. Measuring the impact of manganese exposure on children's neurodevelopment: advances and research gaps in biomarker-based approaches. Environ Health. 2016;15(1):91.

86. Eastman RR, Jursa TP, Benedetti C, Lucchini RG, Smith DR. Hair as a biomarker of environmental manganese exposure. Environ Sci Technol. 2013:47(3):1629-37.

87. Ward EJ, Edmondson DA, Nour MM, Snyder S, Rosenthal FS, Dydak U. Toenail manganese: a sensitive and specific biomarker of exposure to manganese in career welders. Ann Work Expos Heal. 2017;62(1):101-11. 
88. Smith D, Gwiazda R, Bowler R, Roels H, Park R, Taicher C, et al. Biomarkers of Mn exposure in humans. Am J Ind Med. 2007;50(11):801-11.

89. Niedzielska K, Struzak-Wysokińska M, Wujec Z. Analysis of correlations between the content of various elements in hard tissues of milk teeth with and without caries. Czas Stomatol. 1990;43(6):316-22.

90. Zheng W, Fu SX, Dydak U, Cowan DM. Biomarkers of manganese intoxication. Neurotoxicology. 2011;32(1):1-8.

91. Lucas EL, Bertrand P, Guazzetti S, Donna F, Peli M, Jursa TP, et al. Impact of ferromanganese alloy plants on household dust manganese levels: implications for childhood exposure. Environ Res. 2015;138:279-90.

92. Ntihabose R, Surette C, Foucher D, Clarisse O, Bouchard MF. Assessment of saliva, hair and toenails as biomarkers of low level exposure to manganese from drinking water in children. Neurotoxicology. 2018;64:126-33.

93. Wang D, Du X, Zheng W. Alteration of saliva and serum concentrations of manganese, copper, zinc, cadmium and lead among career welders. Toxicol Lett. 2008;176(1):40-7.

94. Laohaudomchok W, Lin X, Herrick RF, Fang SC, Cavallari JM, Christiani DC et al. Toenail, blood, and urine as biomarkers of manganese exposure. J Occup Environ Med. 2011;53(5):506-10.

95. Yaemsiri S, Hou N, Slining MM, He K. Growth rate of human fingernails and toenails in healthy American young adults. J Eur Acad Dermatol Venereol. 2010:24(4):420-3.

96. He K. Trace elements in nails as biomarkers in clinical research. Eur J Clin Investig. 2011:41(1):98-102.

97. Rodrigues $E G$, Kile M, Dobson C, Amarasiriwardena C, Quamruzzaman Q Rahman M, et al. Maternal-infant biomarkers of prenatal exposure to arsenic and manganese. J Expo Sci Environ Epidemiol. 2015;25(6):639-48.

98. Arora M, Bradman A, Austin C, Vedar M, Holland N, Eskenazi B, et al. Determining fetal manganese exposure from mantle dentine of deciduous teeth. Environ Sci Technol. 2012;46(9):5118-25.

99. Yoon M, Nong A, Clewell HJ 3rd, Taylor MD, Dorman DC, Andersen ME. Evaluating placental transfer and tissue concentrations of manganese in the pregnant rat and fetuses after inhalation exposures with a PBPK model. Toxicol Sci. 2009;112(1):44-58.

100. Sky-Peck HH. Distribution of trace elements in human hair. Clin Physiol Biochem. 1990;8(2):70-80.

101. Wahlberg K, Arora M, Curtin A, Curtin P, Wright RO, Smith DR, et al. Polymorphisms in manganese transporters show developmental stage and sex specific associations with manganese concentrations in primary teeth. Neurotoxicology. 2018;64:103-9.

102. Kaczkurkin AN, Raznahan A, Satterthwaite TD. Sex differences in the developing brain: insights from multimodal neuroimaging. Neuropsychopharmacology. 2019;44(1):71-85.

103. Ngun TC, Ghahramani N, Sánchez FJ, Bocklandt S, Vilain E. The genetics of sex differences in brain and behavior. Front Neuroendocrinol. 2011;32(2): 227-46.

104. Madison JL, Wegrzynowicz M, Aschner M, Bowman AB. Gender and manganese exposure interactions on mouse striatal neuron morphology. Neurotoxicology. 2011;32(6):896-906.

\section{Publisher's Note}

Springer Nature remains neutral with regard to jurisdictional claims in published maps and institutional affiliations.

Ready to submit your research? Choose BMC and benefit from:

- fast, convenient online submission

- thorough peer review by experienced researchers in your field

- rapid publication on acceptance

- support for research data, including large and complex data types

- gold Open Access which fosters wider collaboration and increased citations

- maximum visibility for your research: over $100 \mathrm{M}$ website views per year

At BMC, research is always in progress.

Learn more biomedcentral.com/submissions 